\title{
FORWARD MODELING OF STANDING KINK MODES IN CORONAL LOOPS. I. SYNTHETIC VIEWS
}

\author{
Ding Yuan ${ }^{1,2,3}$ and Tom VAN Doorsselaere ${ }^{1}$ \\ ${ }^{1}$ Centre for Mathematical Plasma Astrophysics, Department of Mathematics, KU Leuven, Celestijnenlaan 200B bus 2400, \\ B-3001 Leuven, Belgium; DYuan2@uclan.ac.uk \\ 2 Jeremiah Horrocks Institute, University of Central Lancashire, Preston PR1 2HE, UK \\ ${ }^{3}$ Key Laboratory of Solar Activity, National Astronomical Observatories, Chinese Academy of Sciences, Beijing, 100012, China \\ Received 2015 August 7; accepted 2016 March 7; published 2016 April 14
}

\begin{abstract}
Kink magnetohydrodynamic (MHD) waves are frequently observed in various magnetic structures of the solar atmosphere. They may contribute significantly to coronal heating and could be used as a tool to diagnose the solar plasma. In this study, we synthesize the Fe IX $\lambda 171.073 \AA$ emission of a coronal loop supporting a standing kink MHD mode. The kink MHD wave solution of a plasma cylinder is mapped into a semi-torus structure to simulate a curved coronal loop. We decompose the solution into a quasi-rigid kink motion and a quadrupole term, which dominate the plasma inside and outside of the flux tube, respectively. At the loop edges, the line of sight integrates relatively more ambient plasma, and the background emission becomes significant. The plasma motion associated with the quadrupole term causes spectral line broadening and emission suppression. The periodic intensity suppression will modulate the integrated intensity and the effective loop width, which both exhibit oscillatory variations at half of the kink period. The quadrupole term can be directly observed as a pendular motion at the front view.
\end{abstract}

Key words: magnetohydrodynamics (MHD) - Sun: atmosphere - Sun: corona - Sun: oscillations - waves

Supporting material: animations

\section{INTRODUCTION}

In the past decade, significant progress has been achieved in probing the heating and seismological roles of magnetohydrodynamic (MHD) waves in the solar atmosphere (see reviews by Nakariakov \& Verwichte 2005; Liu \& Ofman 2014; Arregui 2015; Jess et al. 2015). Among the MHD wave modes, the slow magnetoacoustic mode propagates anisotropically in a low $\beta$ uniform plasma; the wave energy flows predominantly along the magnetic field line, e.g., De Moortel et al. (2002a, 2002b), Wang et al. (2009a, 2009b), Yuan \& Nakariakov (2012), Kumar et al. (2013, 2015), and Fang et al. (2015). Meanwhile, a fast magnetoacoustic mode could propagate to any direction relative to the magnetic field, i.e., either parallel, perpendicular, or oblique, therefore they are commonly waveguided in a variety of magnetic structures through reflections and refractions. They may couple with Alfvén waves and exhibit mixed wave properties in forms of standing transverse oscillations of coronal loops (Aschwanden \& Schrijver 2011; Nisticò et al. 2013; Verwichte et al. 2013a); large-scale coronal propagating fronts across the whole solar disk (Ofman \& Thompson 2002; Liu et al. 2010; Guo et al. 2015); quasi-periodic fast wave trains along magnetic funnels (Liu et al. 2012; Pascoe et al. 2013; Yuan et al. 2013; Nisticò et al. 2014b); fast wave pulses across randomly structured plasma (Yuan et al. 2015a); ubiquitous propagating kink waves in the entire corona (Tomczyk et al. 2007) and coronal holes (Thurgood et al. 2014; Morton et al. 2015).

Kink waves (the $m=1$ mode, Edwin \& Roberts 1982, 1983; Ruderman 2003; Erdélyi \& Morton 2009; Goossens et al. 2014) were initially observed in active region loops in the Extreme Ultraviolet (EUV) channels of the Transition Region and Coronal Explorer (TRACE, Aschwanden et al. 1999; Nakariakov et al. 1999). The coronal loops were observed to oscillate transversely with amplitudes at a megameter scale in response to flares, i.e., the associated mass ejections (Schrijver et al. 2002; Zimovets \& Nakariakov 2015), filament destabilizations (Schrijver et al. 2002), magnetic reconnection (He et al. 2009), or vortex shedding (Nakariakov et al. 2009). Recently, Nisticò et al. (2013) and Anfinogentov et al. (2013) detected low-amplitude (sub-megameter scale) kink oscillations of coronal loops. Kink waves in this category last for dozens of wave cycles without significant damping, and are apparently not associated with any explosive events (Anfinogentov et al. 2013). Transverse oscillatory motions were also observed in chromospheric spicules (Okamoto \& De Pontieu 2011; Morton 2014), chromospheric mottles (Kuridze et al. 2012), filament threads (Lin et al. 2007, 2009), large prominences (Tripathi et al. 2009; Hershaw et al. 2011; Arregui et al. 2012), polar plumes (Thurgood et al. 2014), coronal rain (Antolin \& Verwichte 2011), helmet streamers (Chen et al. 2010, 2011), and even coronal mass ejections (Lee et al. 2015).

Fundamental (global) standing kink modes are frequently observed in closed coronal loops (Ruderman \& Erdélyi 2009; Van Doorsselaere et al. 2009). The period of coronal transverse waves ranges from 2 to 33 minutes; and the damping time has a similar timescale (Aschwanden et al. 2002; White \& Verwichte 2012). The curved coronal loops are normally assumed to be approximately co-planar; the loop plane intrinsically defines horizontally and vertically polarized kink waves about the loop axis (Ruderman 2009). Horizontal kink waves are more frequently observed, e.g., Nakariakov et al. (1999), Schrijver et al. (2002), Aschwanden et al. (2002), and Zimovets \& Nakariakov (2015), while vertical kink waves were only reported in a limited number of cases, e.g., Wang \& Solanki (2004), Verwichte et al. (2006), Selwa et al. (2007, 2010, 2011), White et al. (2012), and Kim et al. (2014).

The main interest in standing kink modes of coronal loops mainly arises from their role in diagnosing the coronal plasma via MHD seismology (Nakariakov \& Verwichte 2005; De 
Moortel \& Nakariakov 2012). The standing kink mode could be used as a tool to infer magnetic field strength along a coronal loop (Nakariakov \& Ofman 2001; Verwichte et al. 2009, 2010, 2013a). Verwichte et al. (2013b) measured the range of the density contrast and inhomogeneity layer thickness of coronal loops based on the period-damping timescaling law. De Moortel \& Pascoe (2009) are the first to validate MHD seismology with three-dimensional (3D) numerical simulations, and showed that the inverted magnetic field strength agrees with the input magnetic field within a factor of about two. Aschwanden \& Schrijver (2011) and Verwichte et al. (2013a) compared the seismological field and the Alfvéntransit-time-averaged value in the potential field model, and found consistency within an order of magnitude. Chen \& Peter (2015) performed MHD simulations using a realistic coronal model and found that the excited coronal loop oscillations would be effectively used to infer the average magnetic field.

Kink MHD waves are highly incompressible in the long wavelength limit and exhibit only quasi-rigid motions (Goossens et al. 2012). Indeed, in a coronal loop the density (or temperature) perturbation by a kink mode is at the order of $10^{-3}$ or less than the equilibrium value. The observed intensity variations of coronal loops (e.g., O'Shea et al. 2007; Verwichte et al. 2009, 2010) are ascribed to the column depth modulation introduced by the kink motion. Cooper et al. (2003a, 2003b) performed line of sight (LOS) integration through the coronal loop plasma perturbed by MHD waves and demonstrated that intensity modulation could become significant in case of a kink mode, even though the plasma fluid compression is negligible.

Recently, Goossens et al. (2014) showed that the kink mode solution could be decomposed into a quasi-rigid transverse motion and a rotational motion, which is detectable as Doppler velocity oscillations in optically thick lines. That paper confronts interpreting rotational motion as a signature of Alfvén wave (De Pontieu et al. 2012). Therefore, forward modeling would significantly advance the knowledge of kink modes and resolve the dispute on whether a wave with observed rotational motion is a kink or Alfvén wave (e.g., Van Doorsselaere et al. 2008). Moreover, MHD seismology and wave energy estimation strongly rely on correct identification of the wave mode and accurate measurements of wave properties (Goossens et al. 2012; Van Doorsselaere et al. 2014).

Forward modeling is a novel approach that synthesizes the plasma emission observables (Antolin \& Van Doorsselaere 2013; Yuan et al. 2015b). It basically converts analytical or numerical models into observables. Therefore, the inversion process (e.g., MHD seismology, MHD spectroscopy, helioseismology, X-ray tomography), which is originally ill-posed due to the lack of sufficient constraints (or observables), could be better understood in the sense that knowledge of plasma properties is given a priori. Gruszecki et al. (2012) studied the geometric integration of the plasma density of a fast sausage mode of a plasma cylinder. De Moortel \& Bradshaw (2008) demonstrated that the damping rate measured in EUV emission intensity oscillations may not reflect the real damping of MHD waves. Antolin \& Van Doorsselaere (2013) and Antolin et al. (2014) considered the inhomogeneous plasma emission introduced by fast sausage modes and found that the LOS effect and spatial resolution would significantly modify the associated EUV emissions of coronal loops. Yuan et al. (2015b) found that the contribution function of atomic emission (Dere et al. 1997) could cause emission asymmetry for positive and negative temperature perturbations, and could even lead to the detection of half-periodicity.

In this study, we present a forward modeling study of the standing kink modes of coronal loops. Section 2 gives the analytical solution of a kink mode in a coronal loop and the numerical discretization for the forward modeling code. ${ }^{4}$ Section 3 and Section 4 present our results and conclusions, respectively.

\section{MODEL}

\subsection{Standing Kink Mode}

In this paper, we study the standing kink wave in a plasma cylinder embedded in a uniform plasma. The magnetic field is parallel to the axis of the plasma cylinder (i.e., $z$-axis), $\boldsymbol{B}_{0}=B_{0} \hat{z}$. The equilibrium magnetic field $B_{0}$, plasma density $\rho_{0}$, and temperature $T_{0}$ are piecewise functions of the $r$ coordinate:

$$
B_{0}, \rho_{0}, T_{0}=\left\{\begin{array}{l}
B_{\mathrm{i}}, \rho_{\mathrm{i}}, T_{\mathrm{i}}, \text { for } r \leqslant a \\
B_{\mathrm{e}}, \rho_{\mathrm{e}}, T_{\mathrm{e}}, \text { for } r>a,
\end{array}\right.
$$

where $a$ is the radius of the loop. Hereafter, we use the subscripts " $\mathrm{i}$ " and "e" to differentiate the internal and external equilibrium values of the loop system.

The linearized ideal MHD equations give the perturbed quantities that deviate from the magnetostatic equilibrium (see, e.g., Ruderman \& Erdélyi 2009):

$$
\begin{gathered}
\rho_{1}=-\nabla \cdot\left(\rho_{0} \boldsymbol{\xi}\right), \\
\rho_{0} \frac{\partial^{2} \boldsymbol{\xi}}{\partial t^{2}}=-\nabla P_{\mathrm{T} 1}+\frac{1}{\mu_{0}}\left[\left(\boldsymbol{B}_{0} \cdot \nabla\right) \boldsymbol{b}_{1}+\left(\boldsymbol{b}_{1} \cdot \nabla\right) \boldsymbol{B}_{0}\right], \\
\boldsymbol{b}_{1}=\nabla \times\left(\boldsymbol{\xi} \times \boldsymbol{B}_{0}\right), \\
p_{1}-C_{\mathrm{s}}^{2} \rho_{1}=\boldsymbol{\xi} \cdot\left(C_{\mathrm{s}}^{2} \nabla \rho_{0}-\nabla p_{0}\right),
\end{gathered}
$$

where $\boldsymbol{\xi}$ is the Lagrangian displacement vector, $\rho_{0}, p_{0}$, and $\boldsymbol{B}_{0}$ are the plasma density, pressure, and magnetic field in equilibrium, $\rho_{1}, p_{1}$ and $\boldsymbol{b}_{1}$ are the perturbed plasma density, pressure and magnetic field, $P_{\mathrm{T} 1}=p_{1}+\boldsymbol{b}_{1} \cdot \boldsymbol{B}_{0} / \mu_{0}$ is the perturbed total pressure, and $\mu_{0}$ is the magnetic permeability in free space. A few typical speeds are defined to describe the loop system: $C_{\mathrm{s}}=\sqrt{\gamma p_{0} / \rho_{0}}, \quad C_{\mathrm{A}}=B_{0} / \sqrt{\mu_{0} \rho_{0}}, \quad$ and $C_{\mathrm{T}}=C_{\mathrm{A}} C_{\mathrm{s}} / \sqrt{C_{\mathrm{A}}^{2}+C_{\mathrm{s}}^{2}}$ are the acoustic, Alfvén, and tube speed, respectively (Edwin \& Roberts 1983); and $\omega_{\mathrm{s}}=C_{\mathrm{s}} k$, $\omega_{\mathrm{A}}=C_{\mathrm{A}} k$, and $\omega_{\mathrm{T}}=C_{\mathrm{T}} k$ are the corresponding acoustic, Alfvén, and tube frequencies, where $k=\pi n / L_{0}$ is the longitudinal wavenumber, $n$ is the longitudinal mode number $(n=1$ corresponds to the fundamental mode), $L_{0}$ is the length of the loop, and $\gamma=5 / 3$ is the adiabatic index.

The boundary value problem (Equation (2)-(5)) is solved in cylindrical coordinates $(r, \phi, z)$ with the Neumann boundary conditions at $r=a$,

$$
\begin{aligned}
& {\left[P_{\mathrm{T}}\right]_{r=a}=0,} \\
& {\left[\xi_{r}\right]_{r=a}=0,}
\end{aligned}
$$

and the Dirichlet boundary conditions at $r=0, \infty$

$$
\left.P_{\mathrm{T}}\right|_{r=0}<\infty,
$$

\footnotetext{
4 The FoMo code is available at https://github.com/TomVeeDee/FoMo
} 
(a)

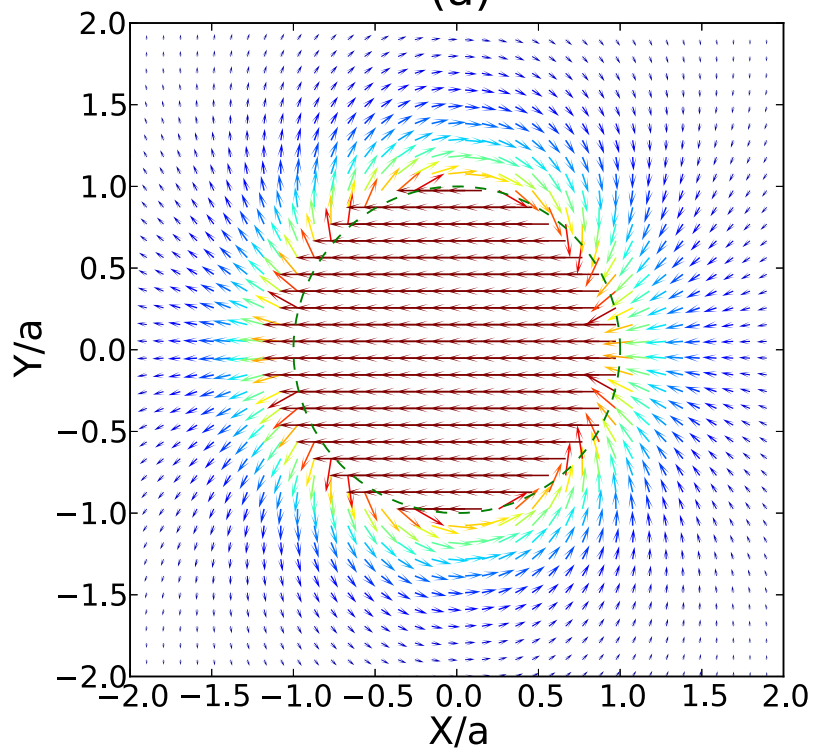

(b)

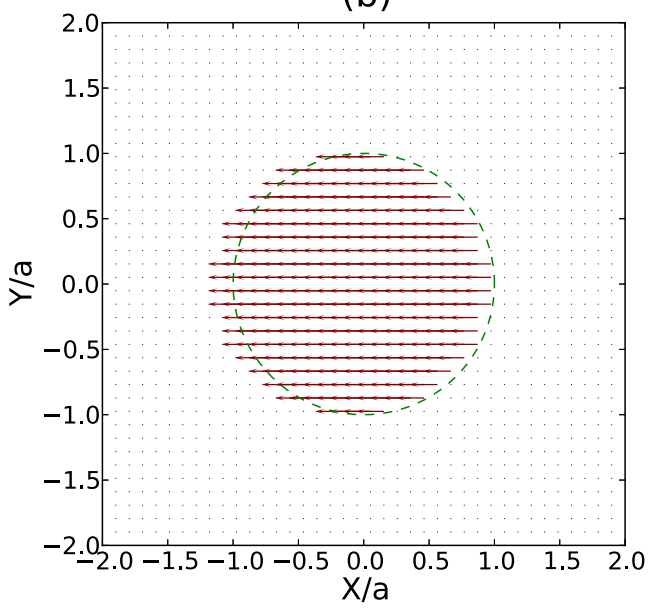

(c)

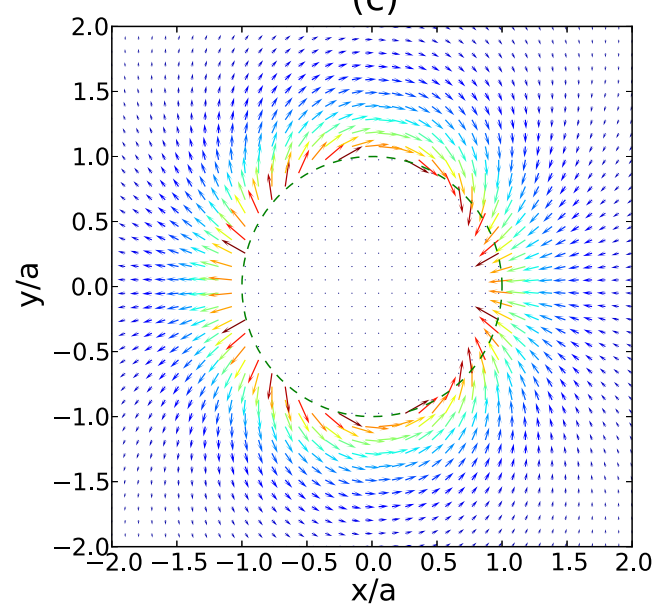

Figure 1. Flow fields at the cross-section of $z=L_{0} / 2$. (a) illustrates the full solution $\left[\tilde{v}_{x}, \tilde{v}_{y}\right]^{\mathrm{T}}$, whereas (b) and (c) show the polarized quasi-rigid motion $\left[\tilde{v}_{x}^{[1]}, \tilde{v}_{y}^{[1]}\right]^{\mathrm{T}}$ and the quadrupole term $\left[\tilde{v}_{x}^{[2]}, \tilde{v}_{y}^{[2]}\right]^{\mathrm{T}}$.

$$
\begin{aligned}
\left.\xi^{2}\right|_{r=0} & <\infty, \\
\left.P_{\mathrm{T}}\right|_{r \rightarrow \infty} & =0, \\
\left.\xi^{2}\right|_{r \rightarrow \infty} & =0,
\end{aligned}
$$

where $P_{\mathrm{T}}$ and $\xi_{r}$ are the total pressure and the radial displacement, respectively. In the case of the standing kink mode $(m=1)$, we Fourier-analyze the perturbed quantities by assuming $P_{\mathrm{T} 1}=A \mathcal{R}(r) \cos (\omega t) \sin (k z) \cos (\phi)$, where $A$ is the amplitude of the perturbed total pressure. The longitudinal profile $\sin (k z)$ ensures that the transverse displacement follows a $\sin k z$-distribution, and therefore has a maximum at the loop apex for the fundamental mode $(n=1)$.

The perturbed total pressure $P_{\mathrm{T} 1}$ (and $\mathcal{R}$ ) must satisfy

$$
\frac{d^{2} P_{\mathrm{T} 1}}{d r^{2}}+\frac{d P_{\mathrm{T} 1}}{r d r}-\left(\kappa_{r}^{2}+\frac{1}{r^{2}}\right) P_{\mathrm{T} 1}=0
$$

where $\kappa_{r}^{2}=\frac{\left(\omega_{\mathrm{s}}^{2}-\omega^{2}\right)\left(\omega_{\mathrm{A}}^{2}-\omega^{2}\right)}{\left(\omega_{\mathrm{s}}^{2}+\omega_{\mathrm{A}}^{2}\right)\left(\omega_{\mathrm{T}}^{2}-\omega^{2}\right)} k^{2}$ is the square of the radial wavenumber and has the dimensionality of wavenumber $k^{2}$. Equation (12) holds for both internal and external plasma, where all quantities are piecewise functions of $r$, and gives $\mathcal{R}=J_{1}\left(\left|\kappa_{r \mathrm{i}}\right| r\right)$ or $K_{1}\left(\kappa_{r \mathrm{e}} r\right)$ for $r<a$ and $r>a$, respectively, where $J_{1}$ and $K_{1}$ are the first order Bessel function of the first kind and the first order modified Bessel function of the second kind, respectively. We redefine $\left|\kappa_{r i}\right|=\sqrt{-\kappa_{r i}^{2}}$, so the dispersion relation for the fast body mode is obtained as

$$
\frac{\kappa_{r \mathrm{e}}}{\rho_{\mathrm{e}}\left(\omega_{\mathrm{Ae}}^{2}-\omega^{2}\right)} \frac{K_{1}^{\prime}\left(\kappa_{r \mathrm{e}} a\right)}{K_{1}\left(\kappa_{r \mathrm{e}} a\right)}=\frac{\left|\kappa_{r \mathrm{i}}\right|}{\rho_{\mathrm{i}}\left(\omega_{\mathrm{Ai}}^{2}-\omega^{2}\right)} \frac{J_{1}^{\prime}\left(\left|\kappa_{r \mathrm{i}}\right| a\right)}{J_{1}\left(\left|\kappa_{r \mathrm{i}}\right| a\right)} .
$$

The perturbed Lagrangian quantities used in the forward modeling code are:

$$
\begin{gathered}
v_{r}=\hat{v}_{r}(r) \sin (\omega t) \sin (k z) \cos \phi, \\
v_{\phi}=\hat{v}_{\phi}(r) \sin (\omega t) \sin (k z) \sin \phi, \\
v_{z}=\hat{v}_{z}(r) \sin (\omega t) \cos (k z) \cos \phi, \\
\rho_{1}=\hat{\rho}_{1}(r) \cos (\omega t) \sin (k z) \cos \phi, \\
T_{1}=\hat{T}_{1}(r) \cos (\omega t) \sin (k z) \cos \phi,
\end{gathered}
$$




\section{(a) Scmi-torus}

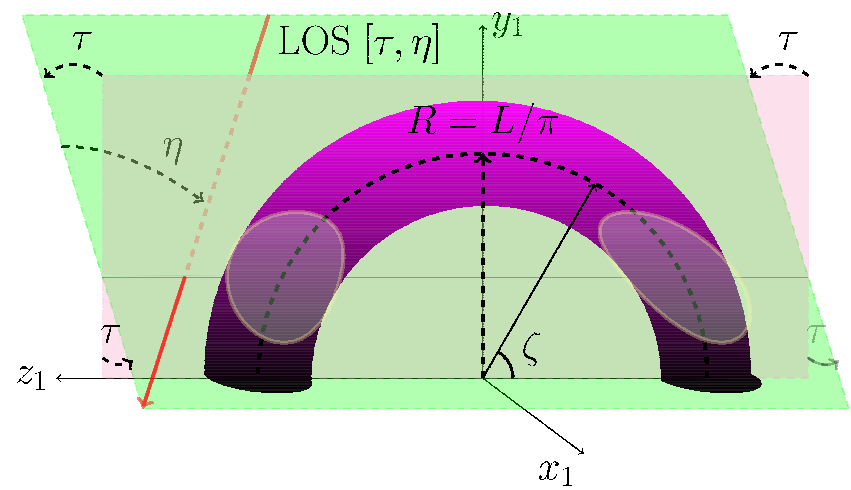

(b) Plasma cylinder

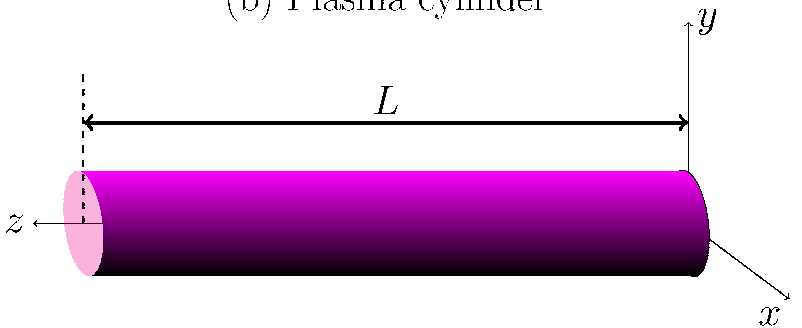

Figure 2. Schematic drawing illustrating how the cylinder is mapped into a semi-torus and the LOS angle definition. The pink plane $\left(y_{1} z_{1}\right.$ plane) is defined by the loop spine. The green plane forms an angle of $\tau$ with the pink plane and their line of intersection is parallel with the $z_{1}$-axis. The LOS is free to vary within the green plane and is quantified by an angle $\eta+\pi / 2$ relative to the $z_{1}$ axis or the line of intersection. An LOS angle is denoted as $[\tau, \eta]$. (a) Top View $\left[0^{\circ}, 0^{\circ}\right]$

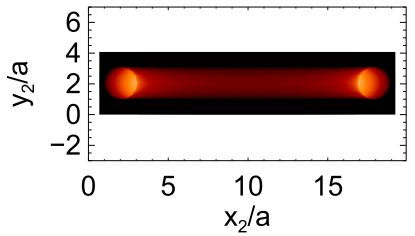

(c) Front View $\left[90^{\circ}, 0^{\circ}\right]$

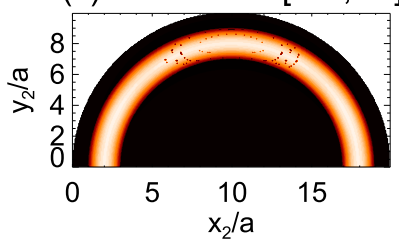

(b) Generic View $\left[45^{\circ}, 45^{\circ}\right]$

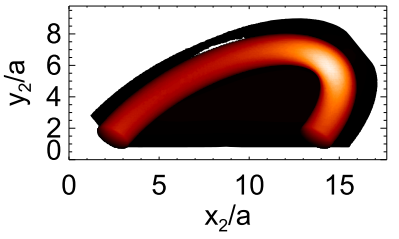

(d) Side View $\left[0^{\circ}, 90^{\circ}\right]$

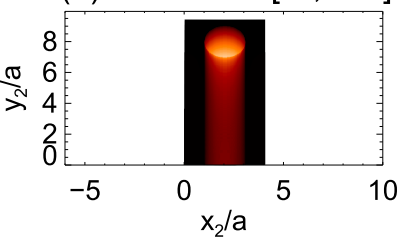

Figure 3. Fe IX $\lambda 171.073 \AA$ synthetic emission at (a) the top view, (b) the generic view, (c) the front view, and (d) the side view. The origins and ranges of the plots are chosen to best match the relative geometries at various views, and will not affect the results at all.

(An animation of this figure is available.)

where

$$
\begin{gathered}
\hat{v}_{r}=-\frac{r d \mathcal{R}}{\mathcal{R} d r} \hat{v}_{0}(r), \\
\hat{v}_{\phi}=\hat{v}_{0}(r)=\frac{A \mathcal{R} \omega}{r \rho_{0}\left(\omega^{2}-\omega_{\mathrm{A}}^{2}\right)},
\end{gathered}
$$

$$
\begin{gathered}
\hat{v}_{z}=-\frac{C_{\mathrm{T}}^{2} k r}{C_{\mathrm{A}}^{2}} \frac{\left(\omega^{2}-\omega_{\mathrm{A}}^{2}\right)}{\left(\omega^{2}-\omega_{\mathrm{T}}^{2}\right)} \hat{v}_{0}, \\
\hat{\rho}_{1}=\frac{\left(\omega^{2}-\omega_{\mathrm{A}}^{2}\right)}{\left(\omega^{2}-\omega_{\mathrm{T}}^{2}\right)} \frac{\rho_{0} r \omega \hat{v}_{0}}{\left(C_{\mathrm{s}}^{2}+C_{\mathrm{A}}^{2}\right)}, \\
\hat{T}_{1}=\frac{\left(\omega^{2}-\omega_{\mathrm{A}}^{2}\right)}{\left(\omega^{2}-\omega_{\mathrm{T}}^{2}\right)} \frac{(\gamma-1) T_{0} r \omega \hat{v}_{0}}{\left(C_{\mathrm{s}}^{2}+C_{\mathrm{A}}^{2}\right)} .
\end{gathered}
$$

The horizontally polarized kink mode has

$$
\begin{aligned}
& v_{x}=\tilde{v}_{x}(r, \phi) \sin (\omega t) \sin (k z), \\
& v_{y}=\tilde{v}_{y}(r, \phi) \sin (\omega t) \sin (k z),
\end{aligned}
$$

where

$$
\tilde{v}_{x}=\hat{v}_{r} \cos ^{2} \phi-\hat{v}_{\phi} \sin ^{2} \phi
$$

$$
\begin{aligned}
& = \begin{cases}v_{00}\left(J_{0}-J_{2} \cos 2 \phi\right), & \text { for } r \leqslant a \\
-\frac{J_{1}^{\prime}\left(\left|\kappa_{r \mathrm{i}}\right| a\right)}{K_{1}^{\prime}\left(\kappa_{\mathrm{re}} a\right)} v_{00}\left(K_{0}+K_{2} \cos 2 \phi\right), & \text { for } r>a,\end{cases} \\
& \tilde{v}_{y}=\hat{v}_{r} \cos \phi \sin \phi+\hat{v}_{\phi} \cos \phi \sin \phi \\
& = \begin{cases}-v_{00} J_{2} \sin 2 \phi, & \text { for } r \leqslant a \\
-\frac{J_{1}^{\prime}\left(\left|\kappa_{r \mathrm{i}}\right| a\right)}{K_{1}^{\prime}\left(\kappa_{\mathrm{re}} a\right)} v_{00} K_{2} \sin 2 \phi, & \text { for } r>a,\end{cases}
\end{aligned}
$$

and $v_{00}=-\frac{A_{\mathrm{i}} \omega\left|\kappa_{r \mathrm{i}}\right|}{2 \rho_{\mathrm{i}}\left(\omega^{2}-\omega_{\mathrm{Ai}}^{2}\right)}$ is the Lagrangian velocity at $r=0$.

We could see that the plasma motion is predominantly polarized along the $x$-direction described by the $J_{0}$ term (also see the Appendix and Goossens et al. 2014). The quadrupole terms $J_{2} \cos (2 \phi)$ and $J_{2} \sin (2 \phi)$ may contribute to the fine structuring of coronal loops associated with kink modes. The vertically polarized transverse mode could be easily obtained by replacing $\phi$ with $\phi+\pi / 2$, while keeping the coordinate system intact.

We rewrite Equations (27) and (29) as

$$
\begin{gathered}
{\left[\begin{array}{c}
\tilde{v}_{x} \\
\tilde{v}_{y}
\end{array}\right]=\left[\begin{array}{c}
\tilde{v}_{x}^{[1]} \\
\tilde{v}_{y}^{[1]}
\end{array}\right]+\left[\begin{array}{c}
\tilde{v}_{x}^{[2]} \\
\tilde{v}_{y}^{[2]}
\end{array}\right],} \\
{\left[\begin{array}{c}
\tilde{v}_{x}^{[1]} \\
\tilde{v}_{y}^{[1]}
\end{array}\right]= \begin{cases}v_{00}\left[\begin{array}{c}
J_{0} \\
0
\end{array}\right], & \text { for } r \leqslant a, \\
-\frac{J_{1}^{\prime}\left(\left|\kappa_{r i}\right| a\right)}{K_{1}^{\prime}\left(\kappa_{\mathrm{re}} a\right)} v_{00}\left[\begin{array}{c}
K_{0} \\
0
\end{array}\right], & \text { for } r>a,\end{cases} } \\
{\left[\begin{array}{c}
\tilde{v}_{x}^{[2]} \\
\tilde{v}_{y}^{[2]}
\end{array}\right]= \begin{cases}-v_{00} J_{2}\left[\begin{array}{c}
\cos 2 \phi \\
\sin 2 \phi
\end{array}\right], & \text { for } r \leqslant a \\
-\frac{J_{1}^{\prime}\left(\left|\kappa_{r i}\right| a\right)}{K_{1}^{\prime}\left(\kappa_{\mathrm{re}} a\right)} v_{00} K_{2}\left[\begin{array}{c}
\cos 2 \phi \\
\sin 2 \phi
\end{array}\right], & \text { for } r>a .\end{cases} }
\end{gathered}
$$

In the thin flux tube limit $(k a \ll 1), J_{0}=1+\mathcal{O}\left((k a)^{2}\right)$ for $r<a$, and $K_{0} / K_{2} \ll 1$ for $r>a$, so the polarized quasi-rigid motion is almost confined within the tube $r<a$ (Figure 1(b)). The quadrupole term is of secondary effect, as $J_{2}=\mathcal{O}\left((k a)^{2}\right)$ for $r<a$, whereas at $r>a, K_{2} / K_{0} \gg 1$, so it dominates the surrounding plasma (Figure 1(c)). But we shall note that the quadrupole term is only a second order term inside the tube, whereas at the ambient plasma, its magnitude is of the first order. 


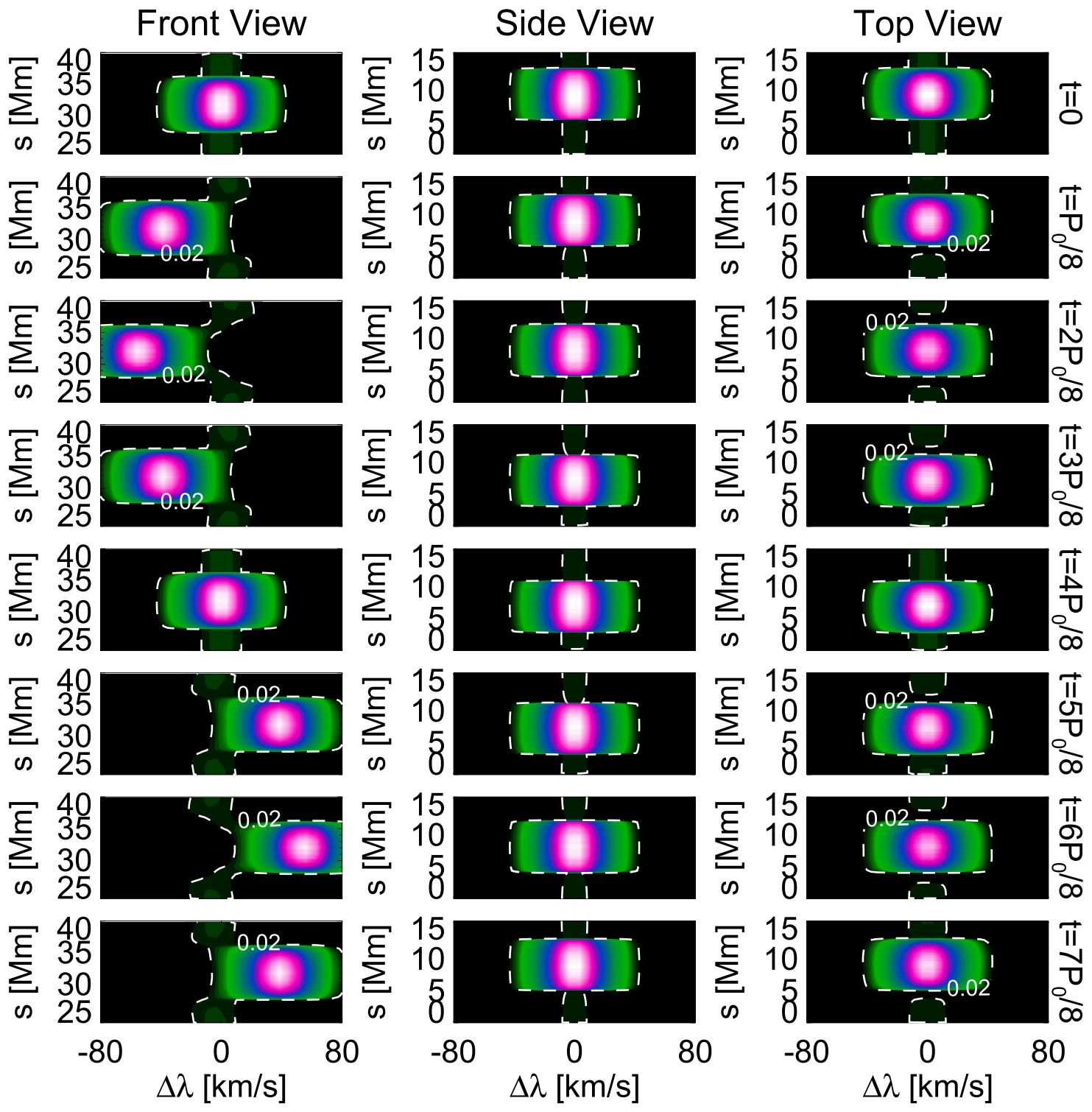

Figure 4. Fe Ix $\lambda 171.073 \AA$ A spectra of sit-and-stare modes along slices $s$ across the loop apex at the front view (left), the side view (middle), and the top view (right). The dashed lines contour the 0.02-level of the peak emission, and highlight the pendular motion at the front view.

(An animation of this figure is available.)

\subsubsection{Correction for Advected Plasma Motion}

Equations (14)-(18) are solutions in Lagrangian coordinates, while we need to synthesize observables at a fixed LOS (Eulerian coordinates), therefore the Lagrangian variables are remapped into Eulerian coordinates. The transverse displacement of a kink mode is of the order of the loop radius $a$ (Aschwanden et al. 2002), and therefore, the advected motion cannot be neglected. The displacement $\boldsymbol{\xi}$ for the plasma fluid at the initial position $[r, \phi, z]$ could be obtained by integrating the velocity with respect to time $t$.

$$
\begin{aligned}
& \xi_{r}=-\hat{v}_{r} / \omega \cos (\omega t) \sin (k z) \cos \phi \\
& \xi_{\phi}=-\hat{v}_{\phi} / \omega \cos (\omega t) \sin (k z) \sin \phi \\
& \xi_{z}=-\hat{v}_{z} / \omega \cos (\omega t) \cos (k z) \cos \phi .
\end{aligned}
$$

In Cartesian coordinates, the displacement is given as

$$
\begin{aligned}
\xi_{x} & =\tilde{\xi}_{x} \cos (\omega t) \sin (k z), \\
\xi_{y} & =\tilde{\xi}_{y} \cos (\omega t) \sin (k z),
\end{aligned}
$$

where $\left[\tilde{\xi}_{x}, \tilde{\xi}_{y}\right]^{\mathrm{T}}=-\left[\tilde{v}_{x}, \tilde{v}_{y}\right]^{\mathrm{T}} / \omega$. Then the new position $[\tilde{x}, \tilde{y}, \tilde{z}]^{\mathrm{T}}$ of the plasma fluid originally at $[x, y, z]^{\mathrm{T}}=$ $[r \cos \phi, r \sin \phi, z]^{\mathrm{T}}$ is

$$
\begin{aligned}
& \tilde{x}(t)=x+\xi_{x}(t), \\
& \tilde{y}(t)=y+\xi_{y}(t), \\
& \tilde{z}(t)=z+\xi_{z}(t) .
\end{aligned}
$$

Thus, the plasma properties (e.g., $\rho_{0}+\rho_{1}$ ) at location $[x, y, z]^{\mathrm{T}}$ will be moved to the position $[\tilde{x}, \tilde{y}, \tilde{z}]^{\mathrm{T}}$. 


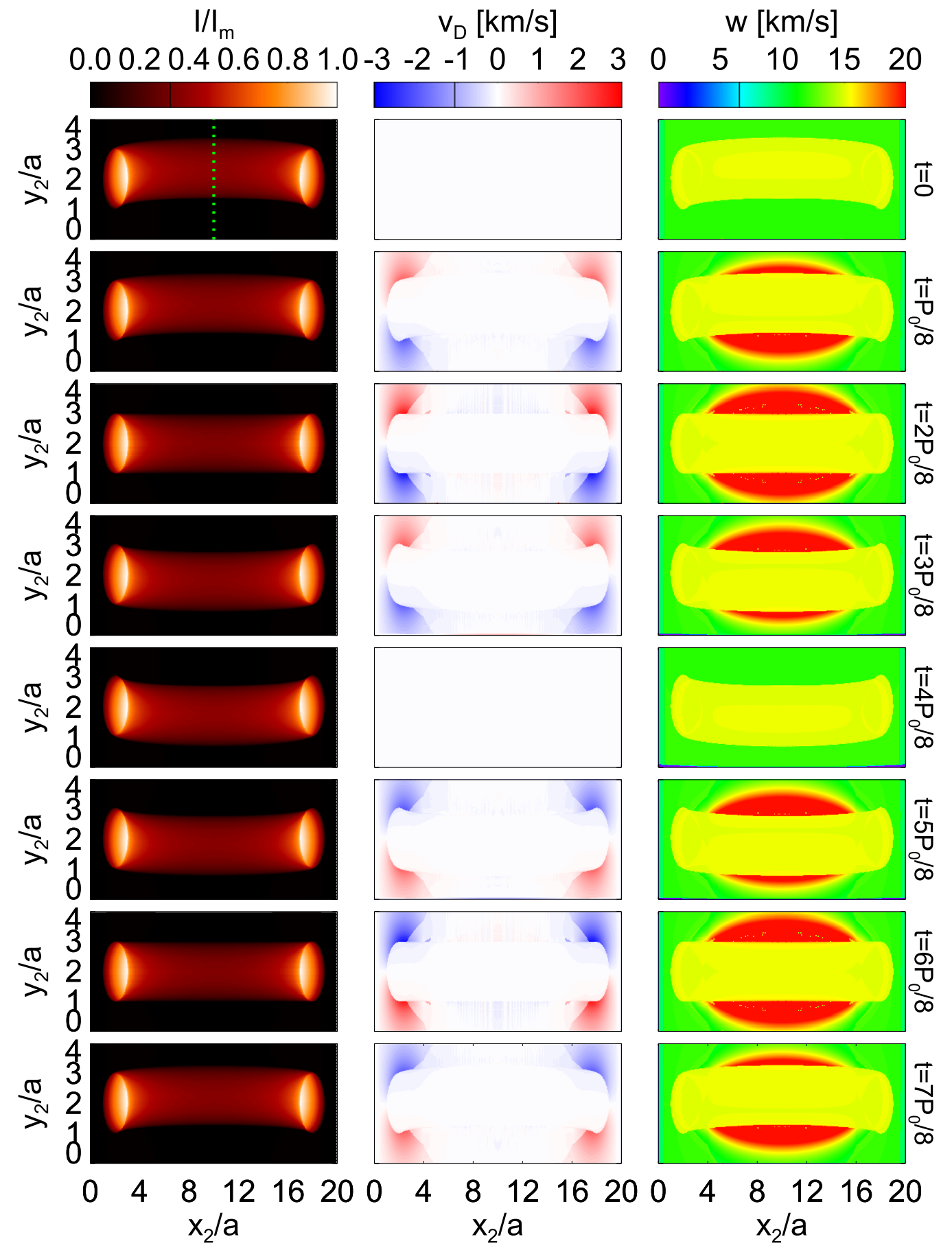

Figure 5. Snapshots of the relative emission $I / I_{m}$ (left), Doppler shift velocity $v_{D}$ (middle), and line width $w$ (right).

(An animation of this figure is available.)

\subsubsection{Mapping into a Semi-torus Structure}

The loop curvature was found to have a secondary effect on the transverse motion of coronal loops (Van Doorsselaere et al. 2009), and therefore we only consider the LOS effect and plasma inhomogeneities by mapping the kink mode solution of a plasma cylinder into a semi-torus structure (see Figure 2). The kink mode displaces the axis of the loop, therefore it has a polarization relative to the plane defined by the static curved loop axis, i.e., the $y_{1} z_{1}$ plane. It is defined as a horizontal kink mode if the loop oscillates out of the $y_{1} z_{1}$ plane (e.g., Aschwanden et al. 1999; Nakariakov et al. 1999). Or otherwise, if the transverse motion of the loop axis is within the $y_{1} z_{1}$ plane, it is termed as a vertical kink mode (e.g., Wang \& Solanki 2004; Verwichte et al. 2006).

After correcting the advected motion, we map the plasma coordinates and the associated plasma parameters into a semitorus structure (Figure 2). The plasma cylinder is bent into a torus within the $y_{1} z_{1}$ plane using the following transform (also 
(a) $I / I_{m}$

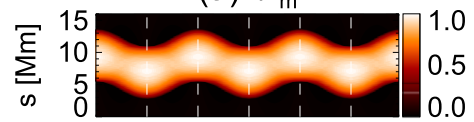

(c) $v_{D}[\mathrm{~km} / \mathrm{s}]$

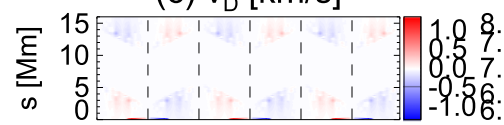

(e) $\mathrm{w}[\mathrm{km} / \mathrm{s}]$

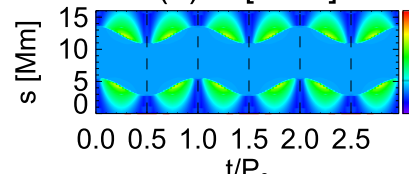

$\mathrm{t} / \mathrm{P}_{0}$ (b) Loop position [Mm]

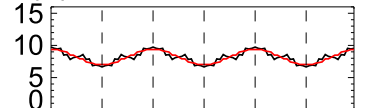

(d) Loop width [Mm]
8.5

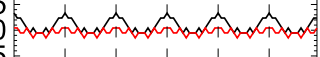
(f) Loop intensity

Figure 6. Sit-and-stare mode across the loop apex at the top view in the Fe IX $\lambda 171.073 \AA$ line and the measurements of loop position, width, and intensity. The red continuous lines plot the corresponding case in the EIS resolution.

see Kuznetsov et al. 2015):

$$
\begin{aligned}
x_{1} & =x, \\
y_{1} & =(R+y) \sin \zeta, \\
z_{1} & =-(R+y) \cos \zeta,
\end{aligned}
$$

where $\zeta=z / R$, and $R=L / \pi$.

The velocity is transformed by

$$
\left[\begin{array}{l}
v_{x_{1}} \\
v_{y_{1}} \\
v_{z_{1}}
\end{array}\right]=\left[\begin{array}{ccc}
1 & 0 & 0 \\
0 & \sin \zeta(z) & \cos \zeta(z) \\
0 & -\cos \zeta(z) & \sin \zeta(z)
\end{array}\right]\left[\begin{array}{l}
v_{x} \\
v_{y} \\
v_{z}
\end{array}\right]
$$

This is basically a rotation of the velocity vector by an angle of $\zeta-\pi / 2$ about the $x$-axis; and $\zeta-\pi / 2$ varies within $[-\pi / 2, \pi / 2]$ for $z \in[0, L]$.

\subsection{Coronal Loop Model}

Coronal loops are highly complex and dynamic structures, observed within a broad range of plasma conditions; see a review by Reale (2014). The loop width varies from a few hundreds (Brooks et al. 2013; Morton \& McLaughlin 2013) to thousands of kilometers (Aschwanden et al. 2002; Aschwanden \& Nightingale 2005; Aschwanden \& Schrijver 2011). A coronal loop may have multi-thermal (Nisticò et al. 2014a), multistranded structures (Peter et al. 2013; Scullion et al. 2014), and may be associated with heating and flows (Winebarger et al. 2002; Klimchuk 2006; Hood et al. 2009). In our study, these fine structures are not considered; and the gravitational stratification is also neglected. Numerical simulations are required to model these features of coronal loop oscillations.

A coronal loop is set up in an equilibrium state. The loop measures $L_{0}=100 \mathrm{Mm}$ in length and $a=4 \mathrm{Mm}$ in radius. The loop density and temperature are $\rho_{\mathrm{i}}=2.5 \cdot 10^{-12} \mathrm{~kg} \mathrm{~m}^{-3}$ $\left(n_{e \mathrm{i}}=1.5 \cdot 10^{9} \mathrm{~cm}^{-3}\right)$ and $T_{i}=0.8 \mathrm{MK}$, respectively. The internal plasma is permeated by a uniform magnetic field $B_{\mathrm{i}}=15 \mathrm{G}$. We choose a density and temperature ratio of $\rho_{\mathrm{i}} / \rho_{\mathrm{e}}=5$ and $T_{\mathrm{i}} / T_{\mathrm{e}}=1.5$, respectively, then the magnetic field strength ratio is obtained by balancing the total pressure at the loop boundary. The plasma beta gives $\beta_{\mathrm{i}}=0.037$ and $\beta_{\mathrm{e}}=0.0048$ for the internal and external plasma, respectively. The corresponding acoustic speeds are $C_{\mathrm{si}}=150 \mathrm{~km} \mathrm{~s}^{-1}$ and $C_{\mathrm{se}}=120 \mathrm{~km} \mathrm{~s}^{-1}$, while the Alfvén speeds are

$C_{\mathrm{Ai}}=840 \mathrm{~km} \mathrm{~s}^{-1}$ and $C_{\mathrm{Ae}}=1900 \mathrm{~km} \mathrm{~s}^{-1}$. These parameters are commonly observed in coronal loops (e.g., Aschwanden \& Boerner 2011; Reale 2014).

For the fundamental mode $(n=1)$, the wavelength is much longer than the loop radius $(k a=0.13)$. The dispersion relationship (Equation (13)) finds a kink mode solution with a period at $P_{0}=3.0$ minute $(\omega=0.034)$. We choose $A_{i}=0.15 \mathrm{~Pa}$, so that the velocity perturbation amplitude is about $55 \mathrm{~km} \mathrm{~s}^{-1}$, and the amplitude of displacement about 1.6 Mm $(0.4 a)$. The kink mode could be considered as highly incompressible (Van Doorsselaere et al. 2008; Goossens et al. 2012); the density (temperature) perturbation is about $0.4 \%$ $(0.3 \%)$ of the equilibrium value. These parameters are commonly observed by the TRACE and SDO/AIA instruments (Aschwanden et al. 2002; Aschwanden \& Schrijver 2011).

\subsection{Forward Model}

The loop system was discretized as given by Equations (14)(18) in Cartesian coordinates. We calculate the plasma properties in a domain of $x(y) \in[-2 a, 2 a]$ and $z \in\left[0, L_{0}\right]$ with $160 \times 160 \times 400$ grid cells. Forward modeling was performed with a fixed output mesh grid $N_{x_{2}} \times N_{y_{2}}=170 \times 340^{5}$ (see details in Yuan et al. 2015b). In contrast to compressive MHD modes (Antolin \& Van Doorsselaere 2013; Reznikova et al. 2014, 2015; Kuznetsov et al. 2015; Yuan et al. 2015b), the kink mode only perturbs the density and temperature to the order of $10^{-3}-10^{-4}$ of the equilibrium values; therefore the effect of the contribution function is of secondary order. The spatial distributions of the plasma properties play a key role in determining the observational features. So we only present the synthetic emission of the Fe IX $\lambda 171.073 \AA$ line; however, the results should be applicable to other optically thin lines. The Fe XII $\lambda 193.509 \AA$ line and the AIA 171 and $193 \AA$ channel were also synthesized, but they only produce redundant results.

The LOS is defined with two independent angles $[\tau, \eta]$ (see illustration in Figure 2), where $\tau$ is the angle between the loop axis plane (pink plane or $y_{1} z_{1}$-plane) and another plane (green plane), which share a line of intersection parallel to the $z_{1}$-axis. The LOS forms an angle of $\eta+\pi / 2$ relative to the $z_{1}$-axis (or the line of intersection). Hereafter, we refer to $\left[0^{\circ}, 0^{\circ}\right]$ as top view, $\left[0^{\circ}, 90^{\circ}\right]$ as side view, $\left[90^{\circ}, 0^{\circ}\right]$ as front view, and $\left[45^{\circ}, 0^{\circ}\right]$ as oblique view for reference. Figure 3 illustrates the synthetic views in the Fe IX $\lambda 171.073 \AA$ line at selected viewing angles, while Figure 4 presents the spectra of the Fe IX $\lambda 171.073 \AA$ line along a slice $s$ perpendicular to the axis of the loop apex at each viewing angle, which is comparable with Figure 9 in Goossens et al. (2014). We note that the loop cross-sectional profile could by approximated by integrating the emissivity along a uniform media $2 \sqrt{a^{2}-r^{2}}$, thus giving a non-Gaussian profile. However, a Gaussian profile is normally assumed and practically observed, e.g., Verwichte et al. (2005), Aschwanden \& Boerner (2011). It implies that coronal loops could be multi-thermal (e.g., Nisticò et al. 2014a), multistranded (Peter et al. 2013), or inhomogeneous (Van Doorsselaere et al. 2004). However, the point-spread function may also play a role, especially in low-resolution instruments. Inhomogeneity in a coronal loop is favored by the resonant absorption theory (Ruderman \& Roberts 2002; Van Doorsselaere et al. 2004; Antolin et al. 2015; Okamoto et al. 2015),

\footnotetext{
5 We refer to the projected output plane as the $x_{2} y_{2}$ plane.
} 


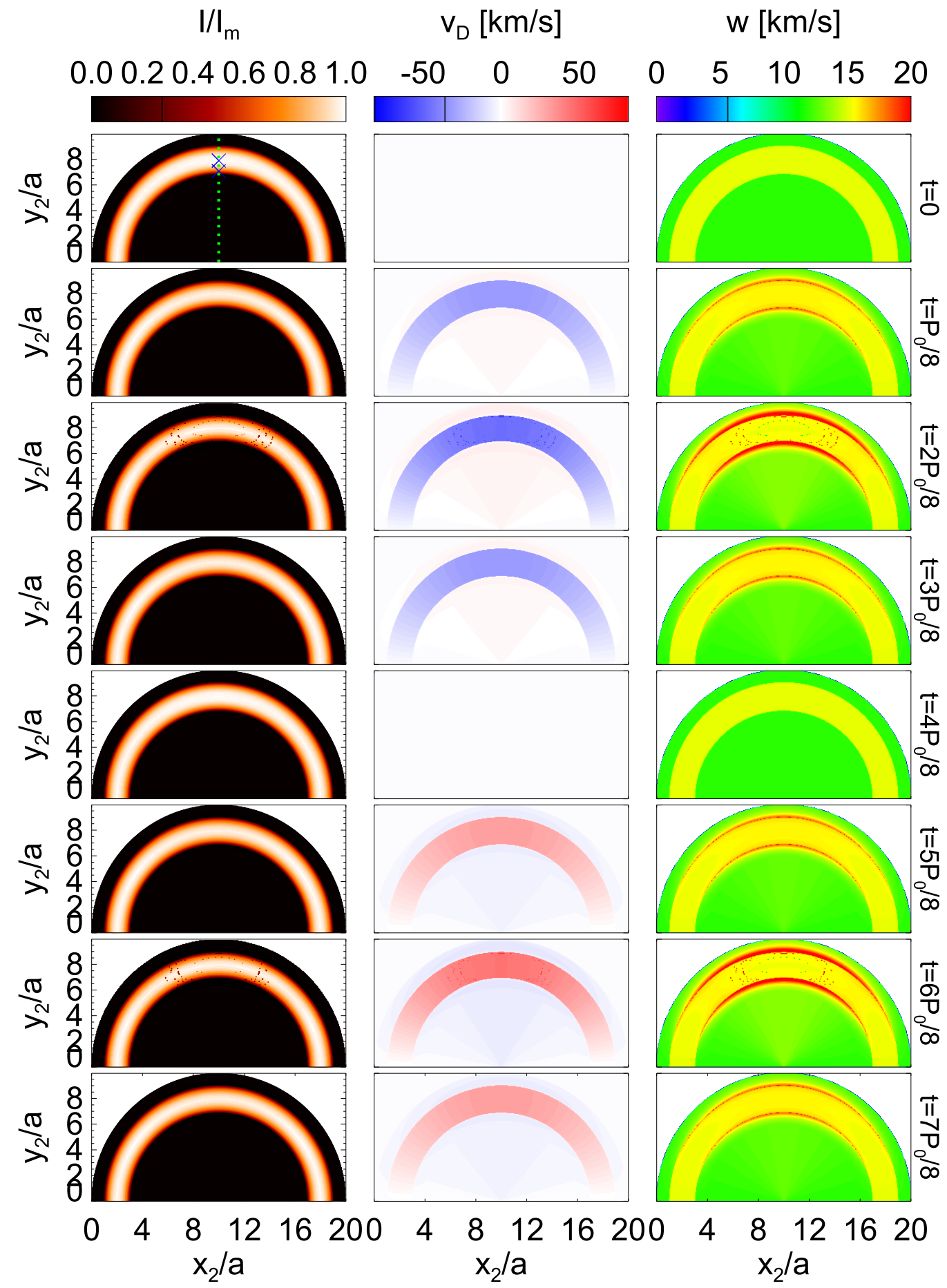

Figure 7. The same as Figure 5 but at the front view. Two crosses mark the positions at $r=0$ and $r=0.8 a$ and the associated dynamic spectra are illustrated in Figure 13.

(An animation of this figure is available.)

which was developed to explain the strong damping of kink waves (Nakariakov et al. 1999). In this study, we do not consider the resonant absorption layer.

\section{RESULTS \\ 3.1. Top View}

Figure 5 presents snapshots of the relative emission intensity $I / I_{m}$, Doppler shift velocity $v_{D}$, and line width $w$ at the top view, where $I_{m}$ is the maximum intensity of the synthetic image series in each viewing angle. At the top view, the loop oscillates within the plane-of-sky; it is clearly seen in the relative intensity, Doppler shift velocity, and line width snapshots. The loop motion is not effectively observed in the Doppler shift, as the plasma motion inside and outside the loop is perpendicular to the LOS.

Figure 6 shows the sit-and-stare mode of a spectrograph, e.g., Hinode/EIS, in the Fe IX $\lambda 171.073 \AA$ line. The time- 
(a) $I / I_{m}$

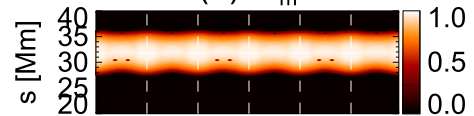

(c) $v_{D}[\mathrm{~km} / \mathrm{s}]$

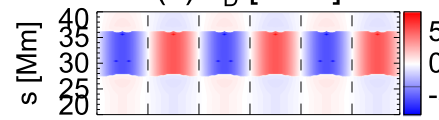

(e) $\mathrm{w}[\mathrm{km} / \mathrm{s}]$

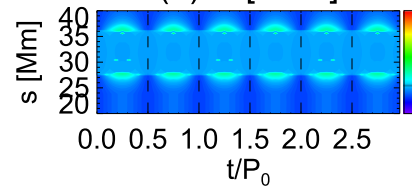

Figure 8. The same as Figure 6 but at the front view.

distance plot (see, e.g., Yuan \& Nakariakov 2012) is taken at a cut perpendicular to the loop axis; see Figure 5. It clearly shows the transverse loop motion with an amplitude of about $1.5 \mathrm{Mm}$ $(0.4 a)$; the associated intensity modulation is about 0.04 . Intriguingly, we also detect loop width (Full-Width at HalfMaximum) variation between 7.0 $\mathrm{Mm}(1.7 a)$ to $7.4 \mathrm{Mm}(1.8 a)$; the amplitude is about $0.2 \mathrm{Mm}$, one order of magnitude smaller than the loop displacement. The periodicity of the loop width and intensity variation is $P_{0} / 2$. The Doppler shift velocity $v_{D}$ is close to zero both inside and outside the loop. Moreover, one could also observe line broadening on the loop periphery. The line width variation has a period of $P_{0} / 2$, and oscillates in phase with the loop intensity variation. Figure 4 (right column) illustrates this effect: the spectrum moves as a whole in space due to the transverse motion; however, the centroid of the spectrum remains unchanged, i.e., $v_{D} \simeq 0 \mathrm{~km} \mathrm{~s}$. At the loop periphery, one could observe significant periodic broadening. The background emission, about $2 \%$ of the loop emission, is associated with the quadrupole terms in Equations (27) and (29) (see the Appendix for derivations).

\subsection{Front View}

At the front view, the transverse motion is along the LOS, so the measured loop displacement is almost zero (Figure 7). The Doppler shift velocity and line width broadening at the loop edges are detectable. The time-distance plot (Figure 8) shows that the loop width oscillates with an amplitude of $0.1 a$ and a period of $P_{0} / 2$. The amplitude (about $0.1 a$ ) observed at the front view is about twice that (about $0.05 a$ ) measured at the top view. Again, we detect line width broadening at the periphery of the loop. This effect may contribute to the non-thermal broadening that has been observed at the edge of active region loops (Doschek et al. 2007). The associated Doppler shift (about $5 \mathrm{~km} \mathrm{~s}^{-1}$ ) of the ambient plasma still exists (Figure 8); however, in contrast to the apparent rotational motion at the top view, the oscillation resembles a pendular motion relative to the loop oscillation. Figure 4 (left column) illustrates this effect: the Doppler shift on the periphery of the loop oscillates in antiphase with the kink motion inside the loop, but with an amplitude of about $10 \%$ of the loop oscillation. This is consistent with Figure 9 in Goossens et al. (2014).

\subsection{Oblique View}

The oblique view (Figure 9) is the most frequently encountered observation on the solar disk. Figure 10 shows the time-distance plot observed in the Fe IX $\lambda 171.073 \AA$ line. Loop oscillation features at the oblique view contain a mixture of the properties observed at the top and front views: loop displacement, intensity modulation, and loop width vary at moderate levels.

\subsection{Side View}

The side view and its variations are the most probable viewing angles for off-limb coronal loops; see, e.g., Verwichte et al. (2004). Figure 11 displays a complete cycle of the standing kink wave at the side view. Loop displacement is optimal for observation in the intensity, while the Doppler shift is very small. The line width does not exhibit significant spatial variation over the projected loop. However, line broadening is significantly measurable. The maximum line width broadening is not located at the loop apex; this is because at the apex the plasma motion is almost perpendicular, rather than along the LOS, and the projected fluid motion is only significant at some distance away from the apex.

Figure 12 presents the time-distance plot at the loop apex and the time series of the loop position, width, and intensity variations. The times series of the transverse motion is close to a sinusoidal profile, while in other viewing angles the loop displacement deviates significantly from a harmonic function. We note that at the side view, the loop width measures at $\simeq 8 \mathrm{Mm}$ (about $2 a$ ), whereas other viewing angles normally do not reveal the full width of the loop. The associated loop width and intensity variations are very small.

\section{DISCUSSION AND CONCLUSION}

In this study, we discretized the fundamental standing kink wave solution of a plasma cylinder, corrected for the fluid advection, and mapped the solution into a semi-torus structure to simulate the kink MHD mode of a curved coronal loop. Then we synthesized the EUV emission in the Fe IX $\lambda 171.073 \AA$ line and performed Gaussian fits to the spectra to obtain the observables, i.e., the emission intensity, Doppler shift velocity, and line width.

We find that the cross-sectional intensity distribution of a coronal loop filled with uniform plasma does not follow a Gaussian profile. This means that the complex coronal loop structure has to be considered to fully synthesize loop oscillations. More physics is associated with loop inhomogeneities, i.e., resonant absorption (Ruderman \& Roberts 2002; Van Doorsselaere et al. 2004), phase mixing (Heyvaerts \& Priest 1983), and mode conversion (Pascoe et al. 2010, 2011, 2012).

Loop displacement could be observed in any viewing angle as long as the polarized motion is not along the LOS. This is the intrinsic feature of a kink MHD wave.

Since the density and temperature perturbations are of the order of $10^{-3}-10^{-4}$ of the equilibrium values, the contribution function has a negligible effect on the loop intensity modulation. The kink mode solution could be decomposed into a quasi-rigid transverse motion and a quadrupole term. The quadrupole term appears in both the $v_{x}$ and $v_{y}$ components of the transverse velocity (Equations (27) and (29)). The fluid elements at $[r, \phi]$ and $[r,-\phi]$ (or equally $[r, \phi]$ and $[r, \pi-\phi]$ ) 


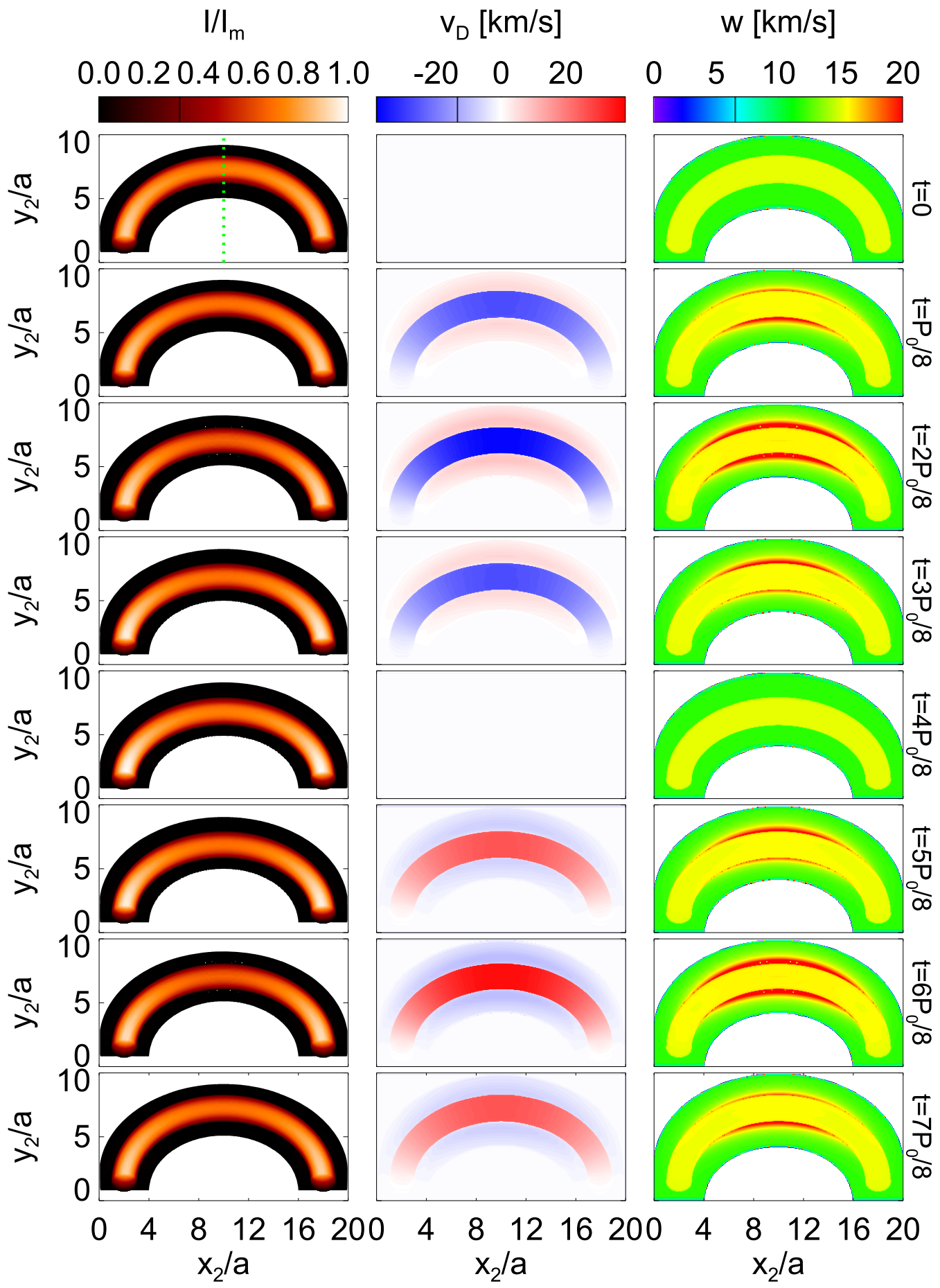

Figure 9. The same as Figure 5, but at the oblique view.

(An animation of this figure is available.)

outside the tube would periodically deform the $r$-shell in the Lagrangian coordinate at the order of $2 \frac{J_{1}^{\prime}\left(\left|\kappa_{\mathrm{r}}\right| a\right)}{K_{1}^{\prime}\left(\kappa_{\mathrm{re}} a\right)} v_{00} K_{2} / \omega$ (see Equations (31) and 32), which is a few percent of the loop radius $a$, if the amplitude of the displacement is close to $a$. Moreover, the fluid elements at $\pm \phi$ of the $r$-shell move to the opposite direction (Figure 14), and thus cause spectral line broadening. The broadening is also accompanied by intensity suppression, as illustrated in Figure 13. At the front view, the emission suppression at $r=0.8 a$ is stronger than that at the loop axis. The quadrupole term effect only becomes significant at the loop edges, where the LOS integrates through more ambient plasma, and has a smaller impact on the spectrum at the loop axis, as the major contributions are from the plasma inside the tube.

Line width broadening is usually measured in the periphery of the loop, where ambient plasma emission is significant. It is associated with the $\cos (2 \theta)$ and $\sin (2 \theta)$ terms in Equations (27) and (29). The line broadening is observed at all views. 
(a) $I / I_{m}$

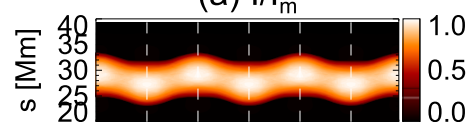

(c) $v_{D}[\mathrm{~km} / \mathrm{s}]$

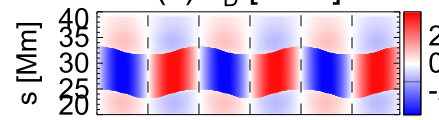

(e) $\mathrm{w}[\mathrm{km} / \mathrm{s}]$

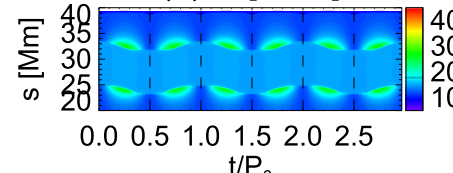

$\mathrm{t} / \mathrm{P}_{0}$ (b) Loop position [Mm]

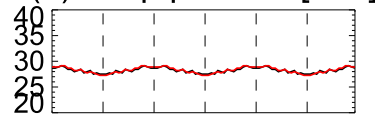

(d) Loop width [Mm]

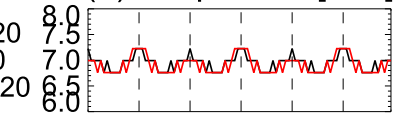

(f) Loop intensity

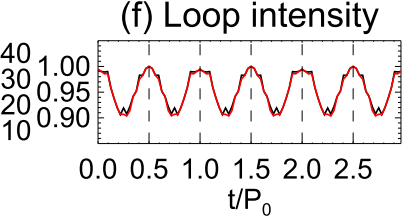

Figure 10. The same as Figure 6 but at the oblique view.

Figure 14 illustrates the reason: at the front view $v_{x}$ could vary from positive to negative along a LOS at loop edge, whereas at the top view $v_{y}$ is anti-symmetric about the $x z$-plane. This is consistent with the case of a vertical transverse wave (Van Doorsselaere \& Nakariakov 2008). Doschek et al. (2007) reported non-thermal broadening at the edge of active region loops, and it may be connected with kink mode perturbations in the loops. However, since there is no report of the associated transverse motion, it may imply that coronal loops have unresolved low-amplitude motions similar to those found by Nisticò et al. (2013) and Anfinogentov et al. (2013).

The intensity modulation at the loop axis is usually detectable. This differs from the findings of Cooper et al. (2003a, 2003b), who only considered static plasma emission. In our study, the spectrum modifications by the MHD wave motions are considered and measured as if they are occurring in realistic observations. This factor could contribute to the integrated LOS intensity variation at the loop axis.

At the front view and its variations, a pendular motion is observed. At the front view, the transverse motion of the loop could be fully observed along the LOS, while the background emission oscillates at an amplitude of a few percent of the loop oscillation amplitude.

It is intriguing that the coronal loop is observed to have an apparent periodic expansion and contraction when undergoing a kink MHD mode wave. The optimal viewing angle to observe this effect is the front view. The amplitude of the loop width variation is about $20 \%-30 \%$ of the transverse loop motion. At the top view, the loop width variation is about half of the amplitude measured at the front view. At the side view, this effect could not be observed. The loop deformation introduced by the quadrupole terms alone is not fully responsible for the loop width variation at such an amplitude. The line width broadening would result in emission intensity suppression at the loop edges, and therefore the effective width of the loop measured in the emission intensity profile is smaller. In such a scenario, we detect effective loop width modulation associated with the periodic redistribution of the intensity across the loop. Aschwanden \& Schrijver (2011) reported loop cross-sectional variations in a vertically polarized standing kink mode and interpreted it as a signature of coupled kink and sausage modes. In our simulation, we predict that the loop width oscillates at a similar amplitude, but with half the period of the kink mode. According to our modeling, Aschwanden \& Schrijver (2011) may have observed an overlap of a steady loop and an oscillating loop of similar density and temperature distribution. Therefore, the loop width variation could be accurately measured. The second paper (Yuan \& Van Doorsselaere 2016) in this series will present the modeling details of this event.

In our loop system, the plasma emission of the coronal loop is about two orders of magnitude larger than the background. If the background emission becomes comparable to that of the loop, the spectroscopic measurement is still valid to some extent (Yuan et al. 2015b). However, one may opt to use another spectral line that is much more sensitive to the plasma emission of interest.

The resolutions of the forward models in each view are better than those offered by current instruments, i.e., Hinode/EIS. Therefore, to predict the possible observations with EIS, we degrade the resolution to an EIS level $\left(1^{\prime \prime}\right)$ by averaging with the neighboring pixel. The red time series in Figures $6,8,10$, and 12 represent the possible sit-and-stare observations with EIS. The loop width is generally measured to be smaller with low-resolution instruments, while the other parameters appear to be a smoothed version of those measured with highresolution instruments, e.g., the SPICE instrument on board the Solar Orbiter.

In this study, we only consider the specific case of a standing kink wave in a coronal loop and synthesize the Fe Ix $\lambda 171.073 \AA$ emissions. However, it should be generally applicable to other optically thin emission lines because in the kink MHD mode, the perturbations to the density and temperature are very tiny. Therefore the spatial distribution of the velocity field plays a determining role in the observational signatures.

The research was supported by an Odysseus grant of the FWO Vlaanderen, the IAP P7/08 CHARM (Belspo), the Topping-Up grant CorSeis, the GOA-2015-014 (KU Leuven), and the Open Research Program KLSA201504 of the Key Laboratory of Solar Activity of National Astronomical Observatories of China (D.Y.). CHIANTI is a collaborative project involving George Mason University, the University of Michigan (USA), and the University of Cambridge (UK).

\section{APPENDIX \\ DERIVATION OF THE QUADRUPOLE TERMS}

Here we demonstrate the derivation of Equations (27) and (29).

$$
\begin{gathered}
\tilde{v}_{x}=\hat{v}_{r} \cos ^{2} \phi-\hat{v}_{\phi} \sin ^{2} \phi \\
=\frac{-A \omega \kappa_{r}}{\rho_{0}\left(\omega^{2}-\omega_{\mathrm{A}}^{2}\right)}\left(\frac{d \mathcal{R}}{\kappa_{r} d r} \cos ^{2} \phi+\frac{\mathcal{R}}{\kappa_{r} r} \sin ^{2} \phi\right) .
\end{gathered}
$$

We define $\lambda=\kappa_{r} r$ and $\mathcal{R}^{\prime}(\lambda)=d \mathcal{R} / d \lambda$. For the plasma motion inside the loop $r<a$ :

$$
\begin{gathered}
\frac{d \mathcal{R}}{\kappa_{r} d r} \cos \phi^{2}+\frac{\mathcal{R}}{\kappa_{r} r} \sin \phi^{2}=J_{1}{ }^{\prime}(\lambda) \cos ^{2} \phi+\frac{J_{1}(\lambda)}{\lambda} \sin ^{2} \phi \\
=\left(\frac{J_{1}}{\lambda}-J_{2}\right) \cos ^{2} \phi+\frac{J_{1}(\lambda)}{\lambda} \sin ^{2} \phi \\
=\frac{J_{1}}{\lambda}-J_{2} \cos ^{2} \phi
\end{gathered}
$$




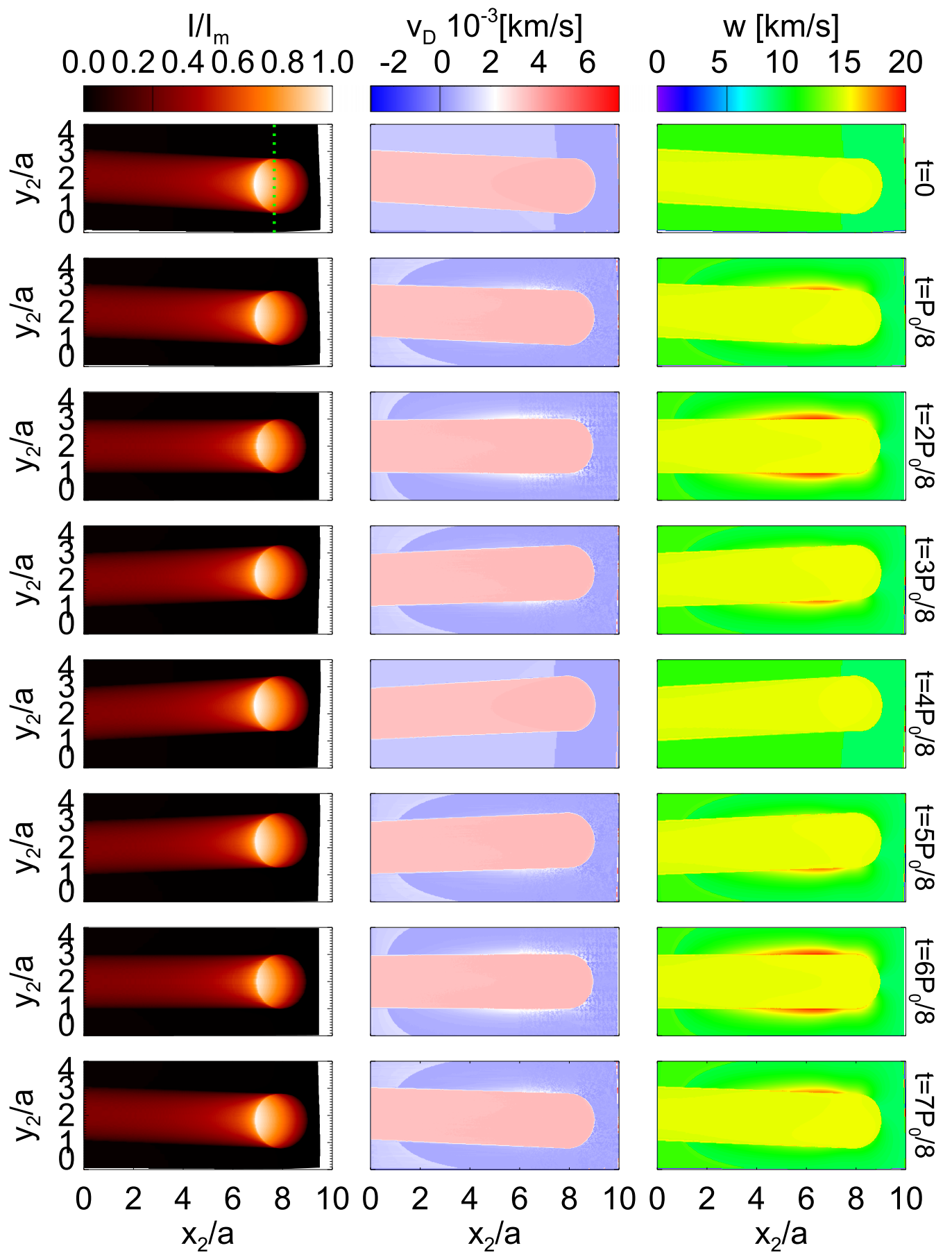

Figure 11. The same as Figure 5 but at the side view.

(An animation of this figure is available.)

$$
\begin{gathered}
=\frac{J_{1}}{\lambda}-\frac{J_{2}}{2}-\frac{J_{2}}{2} \cos 2 \phi \\
=\frac{J_{0}-J_{2} \cos 2 \phi}{2},
\end{gathered}
$$

where we used $J_{1}^{\prime}(\lambda)=J_{1} / \lambda-J_{2}$ and $J_{2}=2 J_{1} / \lambda-J_{0}$ (Olver et al. 2010) in the derivation. We followed the same procedure and used $K_{1}^{\prime}=K_{1} / \lambda-K_{2}$ and $K_{2}=2 K_{1} / \lambda+K_{0}$ (Olver et al. 2010). Then we obtained the plasma motion outside the

$$
\begin{aligned}
& \text { loop } r>a \text { : } \\
& \begin{aligned}
\frac{d \mathcal{R}}{\kappa_{r} d r} \cos \phi^{2}+\frac{\mathcal{R}}{\kappa_{r} r} & \sin \phi^{2}=K_{1}^{\prime}(\lambda) \cos ^{2} \phi+\frac{K_{1}(\lambda)}{\lambda} \sin ^{2} \phi \\
& =\frac{K_{1}}{\lambda}-K_{2} \cos ^{2} \phi \\
& =-\frac{K_{0}+K_{2} \cos 2 \phi}{2} .
\end{aligned}
\end{aligned}
$$


(a) $\mathrm{I} / \mathrm{I}_{\mathrm{m}}$

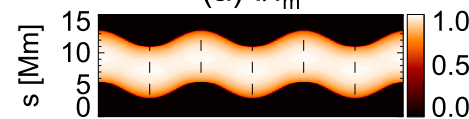

(c) $\mathrm{v}_{\mathrm{D}} 10^{-3}[\mathrm{~km} / \mathrm{s}]$

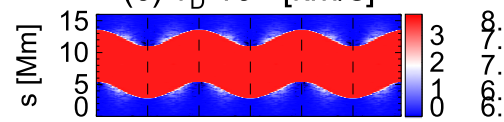

(e) $\mathrm{w}[\mathrm{km} / \mathrm{s}]$

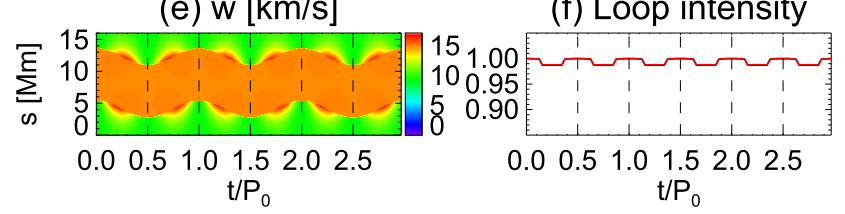

Figure 12. The same as Figure 6 but at the side view.

Here, we obtain the horizontal motion,

$$
\tilde{v}_{x}= \begin{cases}\frac{-A_{\mathrm{i}} \omega\left|\kappa_{r \mathrm{i}}\right|}{2 \rho_{\mathrm{i}}\left(\omega^{2}-\omega_{\mathrm{Ai}}^{2}\right)}\left(J_{0}-J_{2} \cos 2 \phi\right), & \text { for } r \leqslant a \\ \frac{-A_{\mathrm{e}} \omega \kappa_{r \mathrm{e}}}{2 \rho_{\mathrm{e}}\left(\omega^{2}-\omega_{\mathrm{Ae}}^{2}\right)}\left(-K_{0}-K_{2} \cos 2 \phi\right), & \text { for } r>a .\end{cases}
$$
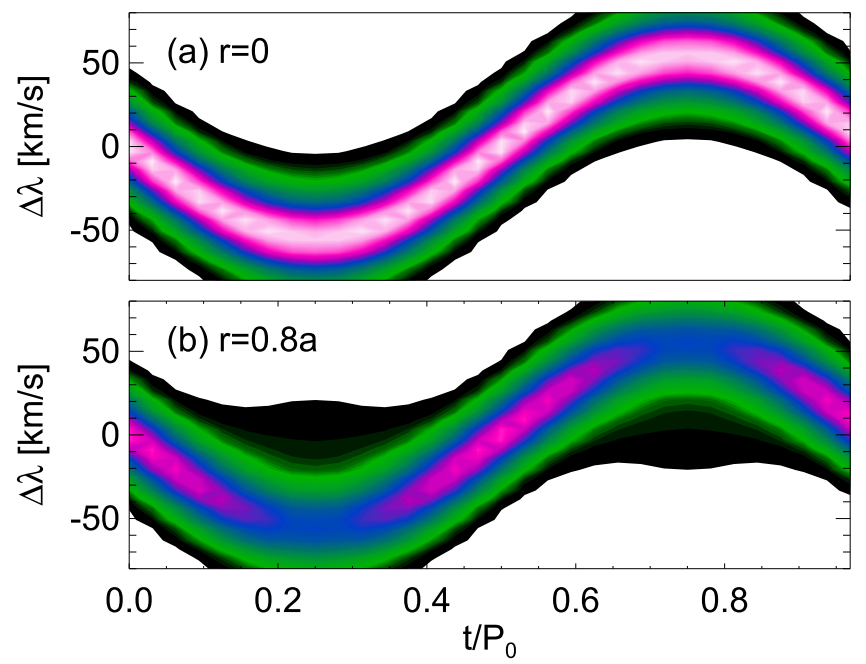

Figure 13. Dynamic spectra extracted at positions $r=0$ (a) and $r=0.8 a$ (b) at the front view, as labeled in Figure 7.

At $r=0, \tilde{v}_{x}=v_{00}=\frac{-A_{\mathrm{i}} \omega\left|\kappa_{r i}\right|}{2 \rho_{\mathrm{i}}\left(\omega^{2}-\omega_{\mathrm{Ai}}^{2}\right.} ;$ while at $r=a$ and $\phi=0$ or $\pi$,

$$
\left.\tilde{v}_{x}\right|_{r=a^{-}}=v_{00}\left(J_{0}\left(\left|\kappa_{r \mathrm{i}}\right| a\right)-J_{2}\left(\left|\kappa_{r \mathrm{i}}\right| a\right)\right),
$$

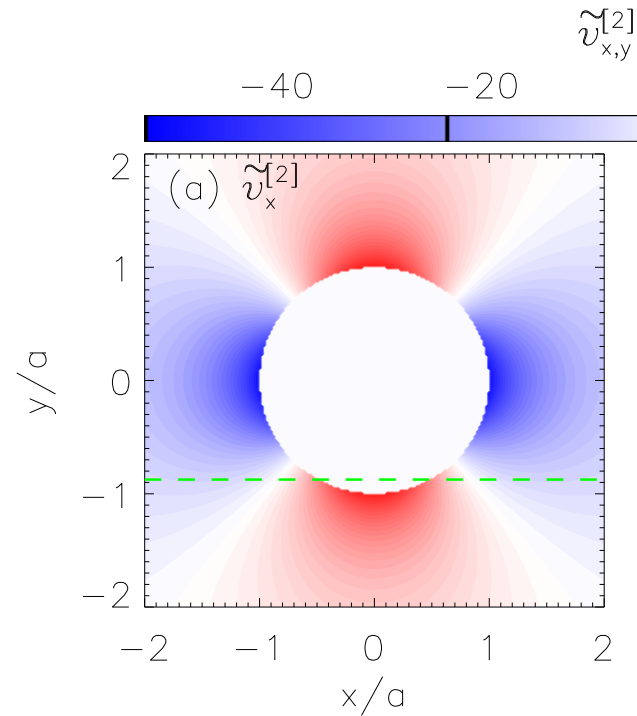

$\widetilde{v}_{x, y}^{[2]}[\mathrm{km} / \mathrm{s}]$

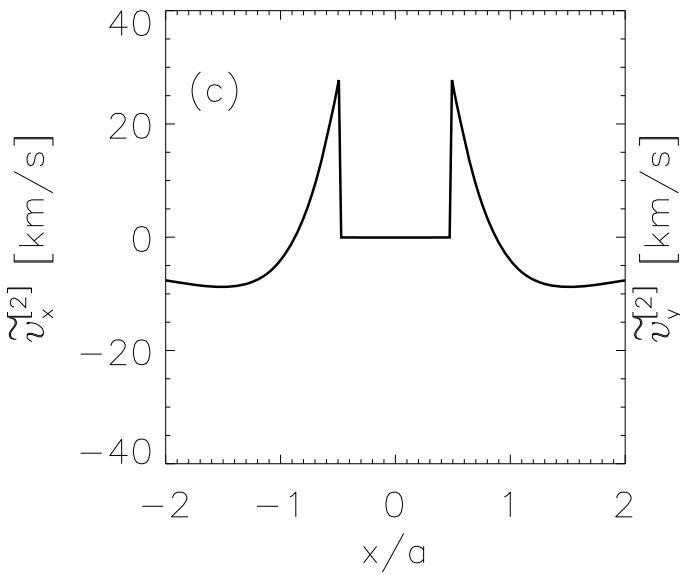

$0 \quad 20 \quad 40$
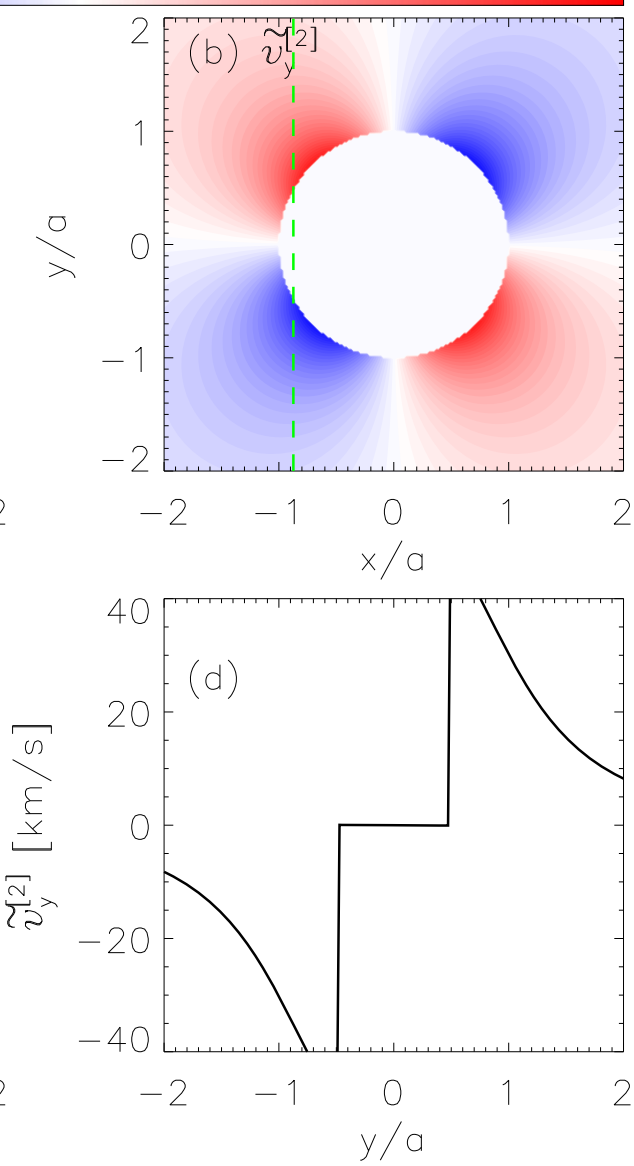

Figure 14. (a) and (b) Cross-sectional distribution of the quadrupole terms $\tilde{v}_{x}^{[2]}$ and $\tilde{v}_{y}^{[2]}$. (c) Profiles of $\tilde{v}_{x}^{[2]}$ along the horizontal dashed line marked in (a). (d) Profiles of $\tilde{v}_{y}^{[2]}$ along the vertical dashed line labeled in (b). 


$$
\begin{aligned}
\left.\tilde{v}_{x}\right|_{r=a^{+}} & =\frac{-A_{\mathrm{e}} \omega \kappa_{r \mathrm{e}}}{2 \rho_{\mathrm{e}}\left(\omega^{2}-\omega_{\mathrm{Ae}}^{2}\right)}\left(-K_{0}\left(\kappa_{r \mathrm{e}} a\right)-K_{2}\left(\kappa_{r \mathrm{e}} a\right)\right), \\
& =\frac{J_{1}^{\prime}\left(\left|\kappa_{r \mathrm{r}}\right| a\right)}{K_{1}^{\prime}\left(\kappa_{r \mathrm{e}} a\right)} v_{00}\left(-K_{0}\left(\kappa_{r \mathrm{e}} a\right)-K_{2}\left(\kappa_{r \mathrm{e}} a\right)\right) .
\end{aligned}
$$

where we recalled the total pressure balance at $r=a$ and the dispersion relationship (Equation (13)):

$$
\begin{aligned}
A_{\mathrm{i}} J_{1}\left(\left|\kappa_{r \mathrm{i}}\right| a\right) & =A_{\mathrm{e}} K_{1}\left(\kappa_{r \mathrm{e}} a\right) \\
\frac{\rho_{\mathrm{i}}\left(\omega_{\mathrm{Ai}}^{2}-\omega^{2}\right) \kappa_{r \mathrm{e}}}{\rho_{\mathrm{e}}\left(\omega_{\mathrm{Ae}}^{2}-\omega^{2}\right)\left|\kappa_{r \mathrm{i}}\right|} & =\frac{J_{1}^{\prime}\left(\left|\kappa_{r \mathrm{i}}\right| a\right) K_{1}\left(\kappa_{r \mathrm{e}} a\right)}{J_{1}\left(\left|\kappa_{r \mathrm{i}}\right| a\right) K_{1}^{\prime}\left(\kappa_{r \mathrm{e}} a\right)} .
\end{aligned}
$$

If we use the relationships $J_{1}^{\prime}=0.5\left(J_{0}-J_{2}\right)$ and $K_{1}^{\prime}=-0.5\left(K_{0}+K_{2}\right)$ (Olver et al. 2010) then we can verify that $\left.\tilde{v}_{x}\right|_{r=a^{-}}=\left.\tilde{v}_{x}\right|_{r=a^{+}}$at $\phi=0$ and $\pi$.

If we follow the same procedure and use the relationships $\left(J_{1}^{\prime}=J_{1} / \eta-J_{2}\right.$ and $K_{1}^{\prime}=K_{1} / \eta-K_{2}$, Olver et al. 2010), then we can easily obtain the vertical component:

$$
\tilde{v}_{y}= \begin{cases}-v_{00} J_{2} \sin 2 \phi, & \text { for } r \leqslant a \\ -\frac{J_{1}^{\prime}\left(\left|\kappa_{r \mathrm{r}}\right| a\right)}{K_{1}^{\prime}\left(\kappa_{\mathrm{re}} a\right)} v_{00} K_{2} \sin 2 \phi, & \text { for } r>a .\end{cases}
$$

\section{REFERENCES}

Anfinogentov, S., Nisticò, G., \& Nakariakov, V. M. 2013, A\&A, 560, A107 Antolin, P., Okamoto, T. J., De Pontieu, B., et al. 2015, ApJ, 809, 72

Antolin, P., \& Van Doorsselaere, T. 2013, A\&A, 555, A74

Antolin, P., \& Verwichte, E. 2011, ApJ, 736, 121

Antolin, P., Yokoyama, T., \& Van Doorsselaere, T. 2014, ApJL, 787, L22

Arregui, I. 2015, RSPTA, 373, 40261

Arregui, I., Oliver, R., \& Ballester, J. L. 2012, LRSP, 9, 2

Aschwanden, M. J., \& Boerner, P. 2011, ApJ, 732, 81

Aschwanden, M. J., de Pontieu, B., Schrijver, C. J., \& Title, A. M. 2002, SoPh, 206, 99

Aschwanden, M. J., Fletcher, L., Schrijver, C. J., \& Alexander, D. 1999, ApJ, 520,880

Aschwanden, M. J., \& Nightingale, R. W. 2005, ApJ, 633, 499

Aschwanden, M. J., \& Schrijver, C. J. 2011, ApJ, 736, 102

Brooks, D. H., Warren, H. P., Ugarte-Urra, I., \& Winebarger, A. R. 2013, ApJL, 772, L19

Chen, F., \& Peter, H. 2015, A\&A, 581, A137

Chen, Y., Feng, S. W., Li, B., et al. 2011, ApJ, 728, 147

Chen, Y., Song, H. Q., Li, B., et al. 2010, ApJ, 714, 644

Cooper, F. C., Nakariakov, V. M., \& Tsiklauri, D. 2003a, A\&A, 397, 765

Cooper, F. C., Nakariakov, V. M., \& Williams, D. R. 2003b, A\&A, 409, 325

De Moortel, I., \& Bradshaw, S. J. 2008, SoPh, 252, 101

De Moortel, I., Hood, A. W., Ireland, J., \& Walsh, R. W. 2002a, SoPh, 209, 89

De Moortel, I., Ireland, J., Walsh, R. W., \& Hood, A. W. 2002b, SoPh, 209, 61

De Moortel, I., \& Nakariakov, V. M. 2012, RSPTA, 370, 3193

De Moortel, I., \& Pascoe, D. J. 2009, ApJL, 699, L72

De Pontieu, B., Carlsson, M., Rouppe van der Voort, L. H. M., et al. 2012, ApJL, 752, L12

Dere, K. P., Landi, E., Mason, H. E., Monsignori Fossi, B. C., \& Young, P. R. 1997, A\&AS, 125, 149

DLMS. 2015, NIST Digital Library of Mathematical Functions, http://dlmf. nist.gov/, Release 1.0.9 of 2014-08-29, online companion to Olver et al. (2010)

Doschek, G. A., Mariska, J. T., Warren, H. P., et al. 2007, ApJL, 667, L109

Edwin, P. M., \& Roberts, B. 1982, SoPh, 76, 239

Edwin, P. M., \& Roberts, B. 1983, SoPh, 88, 179

Erdélyi, R., \& Morton, R. J. 2009, A\&A, 494, 295

Fang, X., Yuan, D., Van Doorsselaere, T., Keppens, R., \& Xia, C. 2015, ApJ, 813,33

Goossens, M., Andries, J., Soler, R., et al. 2012, ApJ, 753, 111

Goossens, M., Soler, R., Terradas, J., Van Doorsselaere, T., \& Verth, G. 2014, ApJ, 788, 9
Gruszecki, M., Nakariakov, V. M., \& Van Doorsselaere, T. 2012, A\&A, 543, A12

Guo, Y., Ding, M. D., \& Chen, P. F. 2015, ApJS, 219, 36

He, J., Marsch, E., Tu, C., \& Tian, H. 2009, ApJL, 705, L217

Hershaw, J., Foullon, C., Nakariakov, V. M., \& Verwichte, E. 2011, A\&A, 531, A53

Heyvaerts, J., \& Priest, E. R. 1983, A\&A, 117, 220

Hood, A. W., Browning, P. K., \& van der Linden, R. A. M. 2009, A\&A, 506, 913

Jess, D. B., Morton, R. J., Verth, G., et al. 2015, SSRv, 190, 103

Kim, S., Nakariakov, V. M., \& Cho, K.-S. 2014, ApJL, 797, L22

Klimchuk, J. A. 2006, SoPh, 234, 41

Kumar, P., Innes, D. E., \& Inhester, B. 2013, ApJL, 779, L7

Kumar, P., Nakariakov, V. M., \& Cho, K.-S. 2015, ApJ, 804, 4

Kuridze, D., Morton, R. J., Erdélyi, R., et al. 2012, ApJ, 750, 51

Kuznetsov, A. A., Van Doorsselaere, T., \& Reznikova, V. E. 2015, SoPh, 290,1173

Lee, H., Moon, Y.-J., \& Nakariakov, V. M. 2015, ApJL, 803, L7

Lin, Y., Engvold, O., Rouppe van der Voort, L. H. M., \& van Noort, M. 2007, SoPh, 246, 65

Lin, Y., Soler, R., Engvold, O., et al. 2009, ApJ, 704, 870

Liu, W., Nitta, N. V., Schrijver, C. J., Title, A. M., \& Tarbell, T. D. 2010, ApJL, 723, L53

Liu, W., \& Ofman, L. 2014, SoPh, 289, 3233

Liu, W., Ofman, L., Nitta, N. V., et al. 2012, ApJ, 753, 52

Morton, R. J. 2014, A\&A, 566, A90

Morton, R. J., \& McLaughlin, J. A. 2013, A\&A, 553, L10

Morton, R. J., Tomczyk, S., \& Pinto, R. 2015, NatCo, 6, 7813

Nakariakov, V. M., Aschwanden, M. J., \& van Doorsselaere, T. 2009, A\&A, 502,661

Nakariakov, V. M., \& Ofman, L. 2001, A\&A, 372, L53

Nakariakov, V. M., Ofman, L., Deluca, E. E., Roberts, B., \& Davila, J. M. 1999, Sci, 285, 862

Nakariakov, V. M., \& Verwichte, E. 2005, LRSP, 2, 3

Nisticò, G., Anfinogentov, S., \& Nakariakov, V. M. 2014a, A\&A, 570, A84

Nisticò, G., Nakariakov, V. M., \& Verwichte, E. 2013, A\&A, 552, A57

Nisticò, G., Pascoe, D. J., \& Nakariakov, V. M. 2014b, A\&A, 569, A12

Ofman, L., \& Thompson, B. J. 2002, ApJ, 574, 440

Okamoto, T. J., Antolin, P., De Pontieu, B., et al. 2015, ApJ, 809, 71

Okamoto, T. J., \& De Pontieu, B. 2011, ApJL, 736, L24

Olver, F. W. J., Lozier, D. W., Boisvert, R. F., \& Clark, C. W. (ed.) 2010, NIST Handbook of Mathematical Functions (New York, NY; Cambridge Univ. Press)

O’Shea, E., Srivastava, A. K., Doyle, J. G., \& Banerjee, D. 2007, A\&A, 473, L13

Pascoe, D. J., Hood, A. W., de Moortel, I., \& Wright, A. N. 2012, A\&A, 539, A37

Pascoe, D. J., Nakariakov, V. M., \& Kupriyanova, E. G. 2013, A\&A, 560, A97

Pascoe, D. J., Wright, A. N., \& De Moortel, I. 2010, ApJ, 711, 990

Pascoe, D. J., Wright, A. N., \& De Moortel, I. 2011, ApJ, 731, 73

Peter, H., Bingert, S., Klimchuk, J. A., et al. 2013, A\&A, 556, A104

Reale, F. 2014, LRSP, 11, 4

Reznikova, V. E., Antolin, P., \& Van Doorsselaere, T. 2014, ApJ, 785, 86

Reznikova, V. E., Van Doorsselaere, T., \& Kuznetsov, A. A. 2015, A\&A, 575, A47

Ruderman, M. S. 2003, A\&A, 409, 287

Ruderman, M. S. 2009, A\&A, 506, 885

Ruderman, M. S., \& Erdélyi, R. 2009, SSRv, 149, 199

Ruderman, M. S., \& Roberts, B. 2002, ApJ, 577, 475

Schrijver, C. J., Aschwanden, M. J., \& Title, A. M. 2002, SoPh, 206, 69

Scullion, E., Rouppe van der Voort, L., Wedemeyer, S., \& Antolin, P. 2014, ApJ, 797, 36

Selwa, M., Murawski, K., Solanki, S. K., \& Ofman, L. 2010, A\&A, 512, A76

Selwa, M., Murawski, K., Solanki, S. K., \& Wang, T. J. 2007, A\&A, 462, 1127 Selwa, M., Solanki, S. K., \& Ofman, L. 2011, ApJ, 728, 87

Thurgood, J. O., Morton, R. J., \& McLaughlin, J. A. 2014, ApJL, 790, L2

Tomczyk, S., McIntosh, S. W., Keil, S. L., et al. 2007, Sci, 317, 1192

Tripathi, D., Isobe, H., \& Jain, R. 2009, SSRv, 149, 283

Van Doorsselaere, T., Andries, J., Poedts, S., \& Goossens, M. 2004, ApJ, 606, 1223

Van Doorsselaere, T., Gijsen, S. E., Andries, J., \& Verth, G. 2014, ApJ, 795, 18

Van Doorsselaere, T., \& Nakariakov, V. M. 2008, in ASP Conf. Ser. 397, First Results From Hinode, ed. S. A. Matthews, J. M. Davis, \& L. K. Harra (San Francisco, CA: ASP), 58 
Van Doorsselaere, T., Nakariakov, V. M., \& Verwichte, E. 2008, ApJL, 676, L73

Van Doorsselaere, T., Verwichte, E., \& Terradas, J. 2009, SSRv, 149, 299

Verwichte, E., Aschwanden, M. J., Van Doorsselaere, T., Foullon, C., \& Nakariakov, V. M. 2009, ApJ, 698, 397

Verwichte, E., Foullon, C., \& Nakariakov, V. M. 2006, A\&A, 452, 615

Verwichte, E., Foullon, C., \& Van Doorsselaere, T. 2010, ApJ, 717, 458

Verwichte, E., Nakariakov, V. M., \& Cooper, F. C. 2005, A\&A, 430, L65

Verwichte, E., Nakariakov, V. M., Ofman, L., \& Deluca, E. E. 2004, SoPh, 223, 77

Verwichte, E., Van Doorsselaere, T., Foullon, C., \& White, R. S. 2013a, ApJ, 767,16

Verwichte, E., Van Doorsselaere, T., White, R. S., \& Antolin, P. 2013b, A\&A, 552, A 138

Wang, T. J., Ofman, L., \& Davila, J. M. 2009a, ApJ, 696, 1448
Wang, T. J., Ofman, L., Davila, J. M., \& Mariska, J. T. 2009b, A\&A, 503, L25

Wang, T. J., \& Solanki, S. K. 2004, A\&A, 421, L33

White, R. S., \& Verwichte, E. 2012, A\&A, 537, A49

White, R. S., Verwichte, E., \& Foullon, C. 2012, A\&A, 545, A129

Winebarger, A. R., Warren, H., van Ballegooijen, A., DeLuca, E. E., \& Golub, L. 2002, ApJL, 567, L89

Yuan, D., \& Nakariakov, V. M. 2012, A\&A, 543, A9

Yuan, D., Pascoe, D. J., Nakariakov, V. M., Li, B., \& Keppens, R. 2015a, ApJ, 799, 221

Yuan, D., Shen, Y., Liu, Y., et al. 2013, A\&A, 554, A144

Yuan, D., \& Van Doorsselaere, T. 2016, ApJS, 223, 24

Yuan, D., Van Doorsselaere, T., Banerjee, D., \& Antolin, P. 2015b, ApJ, 807,98

Zimovets, I. V., \& Nakariakov, V. M. 2015, A\&A, 577, A4 